\title{
Self-healing Materials: A Review of Recent Developments
}

Tingting Song, ${ }^{1}$ Bojun Jiang, ${ }^{1}$ Yida Li,${ }^{1}$ Zhuohan Ji, ${ }^{1}$ Hongling Zhou,,${ }^{1}$ Dawei Jiang, ${ }^{1, *}$ Ilwoo Seok, ${ }^{2}$ Vignesh Murugadoss, ${ }^{3}$ Nan Wen ${ }^{1}$ and Henry Colorado ${ }^{4, *}$

\begin{abstract}
Self-healing polymer is a kind of functional polymer materials that can repair scratches, cracks and other mechanical damage, whose unique self-healing ability is of great significance for prolonging the life of materials. Eigen self-healing polymers have become the focus of current research due to their advantages of mild healing conditions and repeatable healing. In this paper, the preparation technology and properties of self-healing elastomers were reviewed, which provided guidance for the preparation of polymers with high repair efficiency and pointed out its future development trend. The self-healing polymer materials based on Diels-Alder reaction, metal bond, hydrogen bond, ionic bond, disulfide bond, etc., are mainly introduced, and their preparation process, healing mechanism and healing properties are reviewed. Although much progress has been made in self-healing elastomers based on different dynamic bonds, the development of materials with high repair efficiency remains a huge challenge. In this paper, various repair pathways of self-healing elastomers were reviewed, which provided guidance for the balance between repair and mechanical properties. The development of inherently self-healing polymers was also prospected.
\end{abstract}

Keywords: Self-healing Polymer; Elastomer; Hydrogen bond; Disulfide bond.

Received: 12 April 2020; Accepted: 6 May 2021.

Article type: Review article.

\section{Introduction}

Materials Science is one of the most fascinating and challenging areas of science, we have evolved from the era of discovering and using natural materials to the epoch of developing and using artificial materials that are more conducive to our production and life, one of which is selfhealing materials. ${ }^{[1-3]}$ Self-healing material is a kind of material which can repair itself when the object is damaged, that can repair the damage caused by long-term mechanical use. Selfhealing materials have the advantages of predominant mechanical properties, ${ }^{[4-6]}$ insulation, heat insulation and high molecular weight, which can be used to simulate the selfhealing phenomenon of organisms, namely, when micro-

\footnotetext{
I College of Chemistry, Chemical Engineering and Resource Utilization, Northeast Forestry University, Harbin 150040, PR China.

${ }^{2}$ Mechanical Engineering, Arkansas State University, PO Box 1740, State University, AR 72467, USA.

${ }^{3}$ Integrated Composites Laboratory (ICL), Department of Chemical Engineering, University of Tennessee, Knoxville TN 37996, USA.

${ }^{4}$ Composites Laboratory, Universidad de Antioquia UdeA, Calle 70 $N^{\circ}$. 52-21, Medellín, Colombia.

*Email: daweijiang@nefu.edu.cn (W. Jiang), henry.colorado@udea.edu.co (H. Colorado)
}

cracks occur, the self-healing materials repair the cracks by the change of the chemical bond or the release and polymerization of the self-healing monomers stored in the materials, thus maintaining the integrity of the matrix structure. In the past, when some materials were damaged, they had to be discarded and replaced with new materials, resulting in waste. In addition, regular inspections and maintenance also require significant costs, but self-healing materials can effectively address this aspect. In consequence, compared with traditional materials, self-healing materials have excellent performance and a wider use of the environment.

Although the idea of a self-healing material dates back to the mid-20th century, its first success came in 2001, when Wyett et al. experimented with microcapsules ${ }^{[7,8]}$ embedded in the composite containing a healing agent, its cracking caused the healing agent to disperse, interact with the catalyst, and polymerize in the composite. This concept was then further developed to study the method of extending the fatigue life of the self-healing properties in the uniform system and to obtain up to $90 \%$ of the restored fracture toughness. Chen and his colleagues had developed an organic polymer material ${ }^{[9]}$ that can be heated at temperatures above 120 degrees Celsius and then recoated, which has the advantage of not needing a catalyst. Whereas, all these methods are only suitable for preventing macro-cracks, that is, catastrophic failure, and 


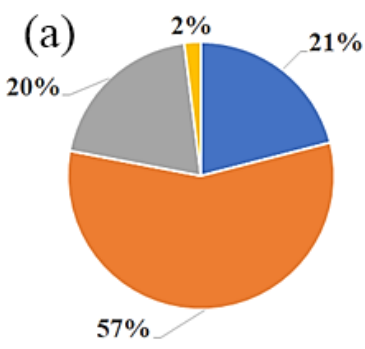

घ Consumer goods

n car

a building

a Aeronautics and astronautics

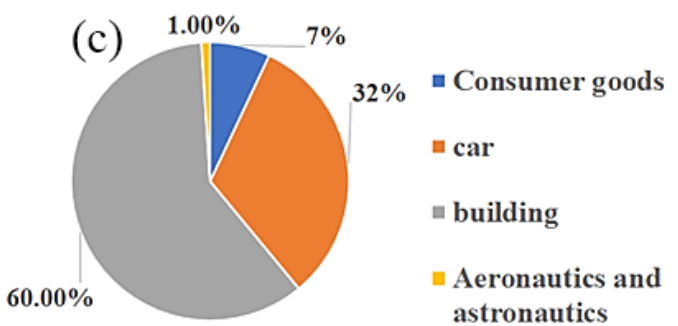

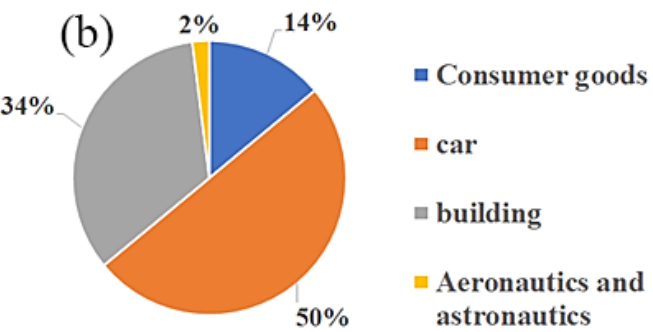

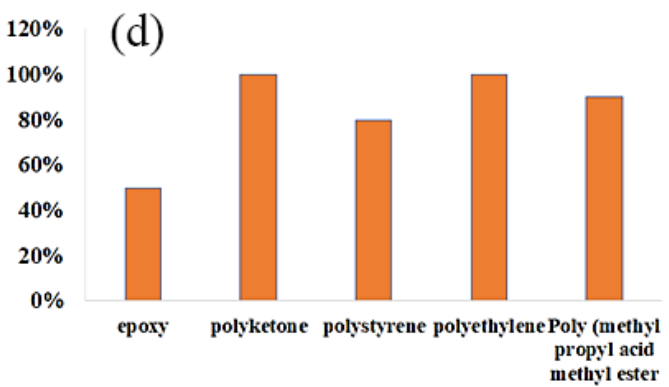

Fig. 1 The proportion of the output value of different fields of self-healing in 2018 (a), 2019 (b), and 2020 (c), respectively. (d) The self-healing efficiency of different repair systems reflected by DA.

hardly any effect on distributed damage.

Last several years, the importance of self-healing materials had been gradually recognized, and its mechanical properties, ${ }^{[10-13]}$ energy absorption and conversion efficiency, and self-healing properties had yet to be further improved in scientific research and practical applications. For decades, scientists and engineers had invested heavily in developing self-healing materials to improve the safety, longevity, energy efficiency and environmental impact of man-made materials. Until now, the research on self-healing materials is still in full swing.

Self-healing materials are widely used in military equipment, electronic products, ${ }^{[14-19]}$ automobiles, airplanes, ${ }^{[20-}$ 22] building materials, ${ }^{[23]}$ biological materials, ${ }^{[24]}$ medical materials ${ }^{[25-29]}$ and biomimetic technology, ${ }^{[30,31]}$ The proportion of application is also increasing with each passing year (Figs. $1 \mathrm{a}, \mathrm{b}$ and $\mathrm{c}$ ), for instance, self-healing materials can be used in dentistry in medical field. With its superior self-healing performance, the service life of materials can be prolonged and the integrity of body structure can be maintained. Selfhealing materials also play an important role in medical hydrogels, ${ }^{[32,33]}$ some self-healing hydrogels can be used for the culture of neural stem cells precursor cells, and some selfhealing hydrogels have temperature and $\mathrm{PH}$ responses, it has great potential application value in biological science and biotechnology related fields. In the meantime, self-healing materials can also be used on smartphones and tablet screens in electronic products. Most importantly, they can improve the service life of mobile phones and tablets, reducing the waste of resources and fu4-3nds. Self-healing materials have tremendous potential applications for electronic skin, ${ }^{[34-36]}$ energy storage devices, sensors, ${ }^{[37]}$ conductive coatings and supercapacitors. Therefore, it is particularly significant to understand the classification and characteristics of self- healing materials, repair systems (Fig. 1d) and characteristics of different self-healing materials.

The development, types, application fields and characteristics of self-healing materials are reviewed in this paper. The dynamic reversible bond self-healing in eigen-type self-healing is mainly introduced, which key self-healing is subdivided. Self-healing materials based on reversible disulfide bond, hydrogen bond, metal coordination bond, ionic bond and Diels-Alder reaction are introduced, while the latest development and characteristics of miscellaneous self-healing materials are also introduced. According to the special properties of different self-healing materials, the future application of these materials is prospected.

\section{Classification and Properties}

\subsection{Self-healing mechanism based on reversible disulfide bonds}

In research work, we classify the concept of self-healing, which is based on the use of disulfide bonds between rubber networks that can fully recover their mechanical properties at moderate temperatures, the key to which is the exchange of disulfide bonds. ${ }^{[38,39]}$

Based on the characteristics of reversible repair and mild repair condition of disulfide bond, introducing reversible disulfide bonds with elastomers to prepare self-healing materials are widely used. Nevertheless, there are few studies on the self-healing properties of materials based on disulfide bond exchange reaction, especially on the self-healing kinetics of coatings under Ultra Violet (UV) light ${ }^{[40,41]}$ and heating conditions. Recently, Zhao ${ }^{[42]}$ introduced a disulfide bond between UV-Curable polyurethane acrylic resins, demonstrating that cracks in the cured resin material can be repaired to UV light. First and foremost, UV curable polyurethane acrylic resin (DSPUA) with disulfide bond was 
(a)

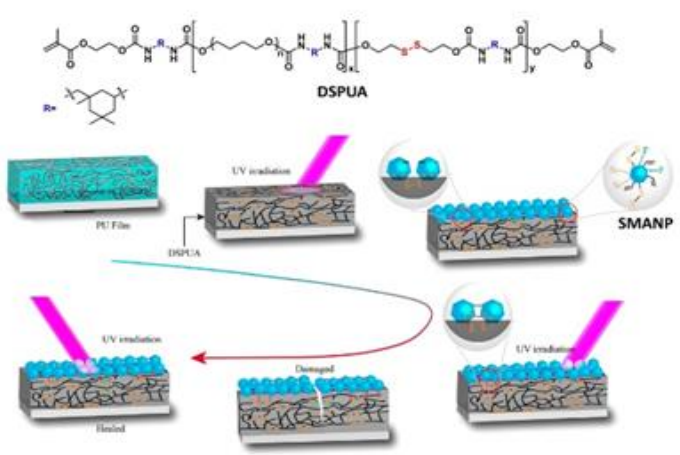

(b)

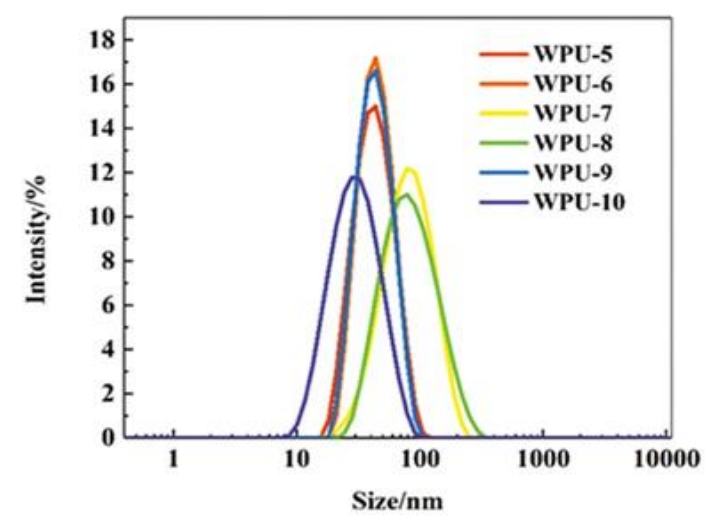

Fig. 2 (a) The structural formula of DSPUA multifunctional material and the repair and synthesis process pf PU under ultraviolet light. (b) Self-healing ability of WPU composites with different proportions, reproduced with the permission form [46], Copyright 2019 American Chemical Society.

prepared. Furthermore, the surface of $\mathrm{Al}_{2} \mathrm{O}_{3}$ nanoparticles (SMANP) was modified and functioned by hydrophobic fluoroalkyl, organosilane, vinyl and disulfide bonding compounds. Ultimately, the super-hydrophobic coating (SMANP@DSPUA) (Fig. 2a) was prepared by spin-coating SMANP solution to DSPEA substrate and UV curing was carried out. SMANP and DSPEA can be covalently bonded to form rough surfaces with strong micro/nano structures by

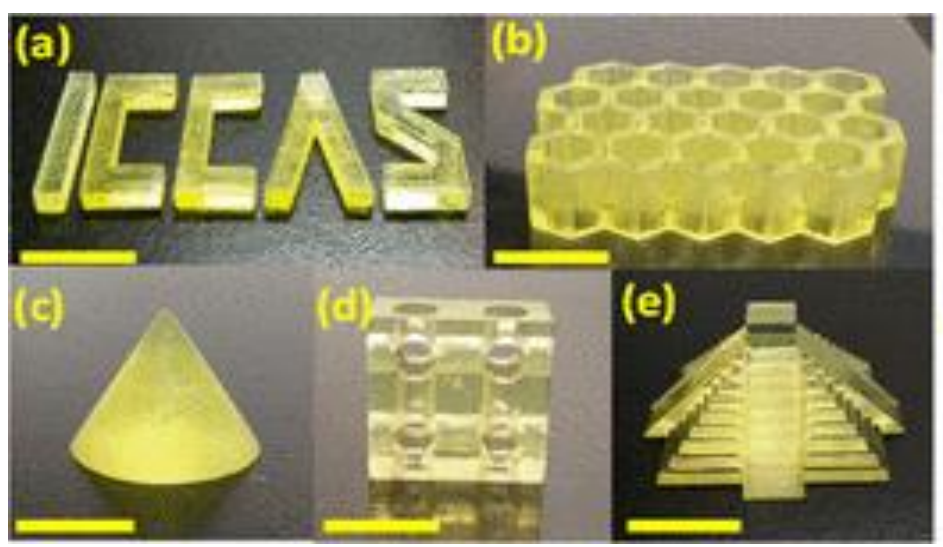

disulfide exchange reaction and ultraviolet curing heated double bond cross-linking. Based on the disulfide exchange reaction, the granular layer and the nascent layer of DSPEA was reformed by covalent bonding with the self-healing process. The fractured surface of the fractured coating could be re-formed into a complete surface, allowing the material to repair itself, even if the wound was sharp. It had been shown that increasing temperature or disulfide bond content could improve the self-healing properties, while UV irradiation could significantly accelerate the self-healing process.

Under UV irradiation, disulfide bonds can be dissolved and cracked, and sulfonyl radicals can be formed, which photochemical process can effectively generate and reconstitute sulfhydryl groups by controlling the UV conditions, thus accelerating the disulfide bond exchange reaction. Under $15 \mathrm{mw} / \mathrm{cm}^{2}$ UV irradiations, the SMANP@DSPUA coating was cut off, the fracture surface of the damaged coating was stored at $80^{\circ} \mathrm{C}$, and under different UV irradiation time, the coating can self-heal to form a complete surface after $30 \mathrm{~min}$ of irradiation, which indicates that the double sulfur bond self-healing material can self-heal faster under UV condition. The results showed that high temperature or high disulfide bond content can significantly improve the self-healing properties. Most importantly, UV irradiation can greatly improve the self-healing rate of the coating, which is due to the generation and reorganization of sulfanilamide group, which provides a great possibility for the self-healing of the coating in sunlight.

Furthermore, the self-healing mechanism of microcracks on waterborne polyurethane ${ }^{[3-45]}$ (WPU) (Fig. 2b) with different disulfide bond contents had been studied in detail by Zhang. ${ }^{[46]}$ The Gel Permeation Chromatography (GPC) was used to calculate the exchange reaction activation energy of disulfide bond between $20.42 \mathrm{~kJ} / \mathrm{mol}$. The healing rate of SHWPUs (Synthesis of Self-Healing Waterborne Polyurethane) was $100 \%$ within 15 min at $75{ }^{\circ} \mathrm{C}$. compared with traditional self-healing materials, SHWPUs had more complete healing effect and milder environment.

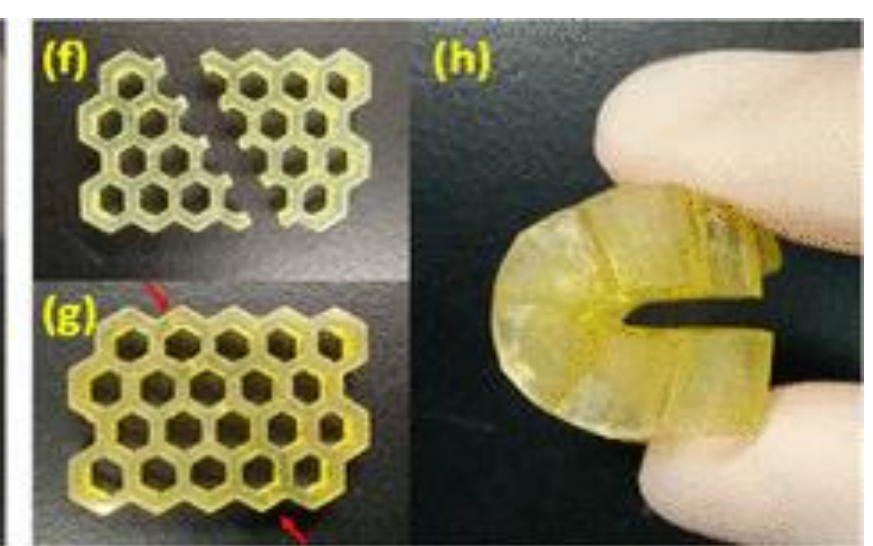

Fig. 3 A 3D object made by DLP 3D printing of the photon-induced polymer PUA-N1 (a) ICCAS, (b) honeycomb structure made by 3D printing (c) a cone, (d) a hollow cube, and (e) a pyramid. ( F-h) Honeycomb structure self-healing experiment. The honeycomb structure was cut into two parts, which were cured at $80^{\circ} \mathrm{C}$ for $12 \mathrm{~h}$. After curing, the sample could be freely bent, that is, the material completed self-healing, reproduced with the permission from [49], Copyright 2019 American Chemical Society. 
Disulfide bonds can also be combined with 3D printing, ${ }^{[47,48]} \mathrm{Li}^{[49]}$ used DLP 3D Printing technology to prepare self-healing polyurethane elastomer. To begin with, a kind of polyurethane acrylate containing disulfide bond was synthesized, in addition, the photo initiator and acrylic acid were mixed by ethyl as reaction diluent to obtain the photopolymer resin. Light curing resin curing speed, advantageous processing performance, DLP 3D printing can be used to print the size of high precision objects. Compared with the traditional casting method, the advantages of 3D printing ${ }^{[50]}$ are time-saving, environmental protection and the ability to produce objects with complex structures. After shearing, joint and healing test, the product sample could be stretched to larger deformation, the tensile strength and elongation in break of PUA-N1 were $3.39 \pm 0.09 \mathrm{MPa}$ and $400.38 \pm 14.26 \%$ respectively. At $80{ }^{\circ} \mathrm{C}$ for $12 \mathrm{~h}$, the strength of the sample was $3.22 \pm 0.40 \mathrm{MPa}$, and the healing efficiency was about $95 \%$, the tensile strength after the second healing was $2.94 \pm 0.36 \mathrm{MPa}$ and the healing efficiency was about $87 \%$. After the third healing, the tensile strength reached 2.05 $\pm 0.34 \mathrm{MPa}$ and the healing efficiency was about $60 \%$. With the increase in the healing times, the tensile strength and the healing efficiency will decrease. The results showed that the tensile strength decreased from the increase in disulfide bond, on the contrary, the self-healing ability (Fig. 3) increased from the increase in disulfide bond. Therefore disulfide bonds as a relatively stable covalent bond have great potential for the research and application of ester elastomer materials.

\subsection{Self-healing mechanism based on hydrogen bonds}

Hydrogen atoms are bonded with electronegative atoms $\mathrm{X}(\mathrm{N}$, $\mathrm{O}, \mathrm{F}$ ) in the form of hydrogen bonds, as a kind of non-covalent interaction force, hydrogen bond is applied to the research, preparation and method innovation of self-healing materials because of its reversibility and directivity. Inspired by the remarkable capability of self-healing in natural systems, smart self-healing materials are being intensively researched to mimic natural systems to have the ability to partially or completely self-repair damages inflicted on them. ${ }^{[51]}$ Selfhealing mechanisms are classified into extrinsic and intrinsic. ${ }^{[52]}$ In recent years, it is widely used in the synthesis of elastomer self-healing materials and improvement of mechanical properties and self-healing capability of the selfhealing materials, the advantages of its rapid repair, high material sensitivity and wide application range are remarkable.

The self-healing ability and mechanical properties of the self-healing materials can be significantly improved by introducing the hydrogen bond repair mechanism into the original self-healing materials. In self-healing, hydrogen bonds can take the form of single or multiple. Single hydrogen bond has high reversibility, high activity and fast bonding speed, but the interaction of single hydrogen bond is usually too weak to make the material stable enough, so the mechanical properties of self-healing materials cannot be improved. Multiple hydrogen bonds have the advantages of
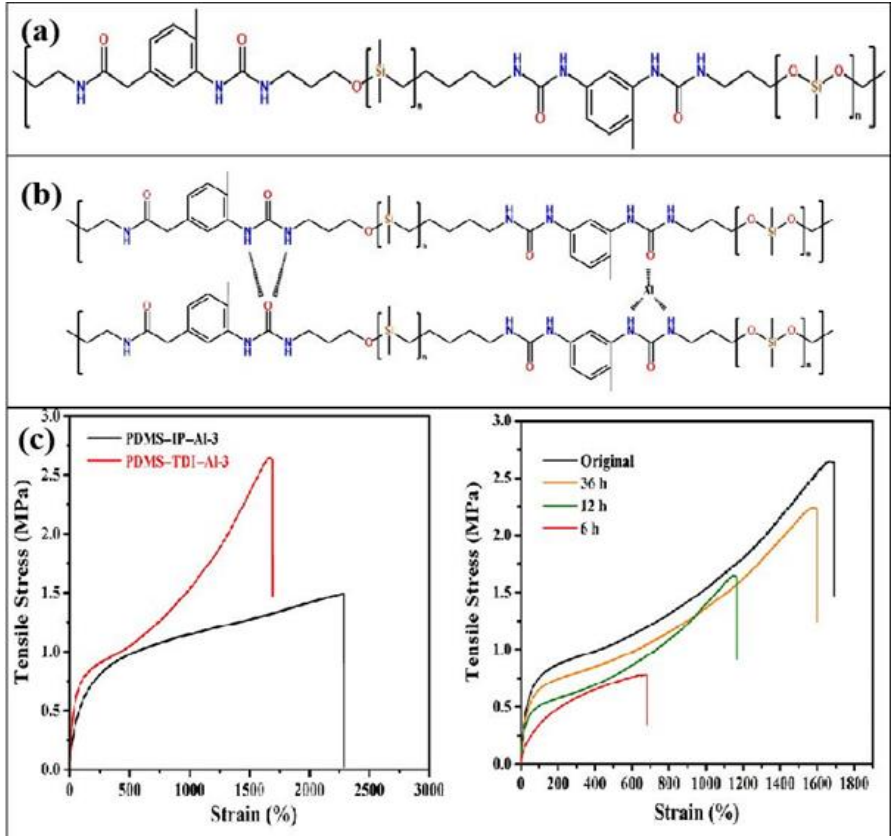

Fig. 4 (a) The chemical structure of PDMS-TDI polymers. (b) A quadruple hydrogen bond formed between PDMS-TDI. (c) Tensile curve with pressure and time of PDMS-TDI-AL-3, reproduced with the permission from [55], Copyright 2019 American Chemical Society.

the above hydrogen bonds, and can also give self-healing materials superior mechanical properties. The deformation of conventional elastomers is dependent on the cross-link density, and there is a trade-off between toughness and stretchability. Therefore, it is a challenge to solve the contradiction between toughness and stiffness. ${ }^{[53]}$ For example, Shao ${ }^{[54]}$ et al. also introduced metal ions (iron ions) to improve mechanical properties while using hydrogen bonds for self-healing. $\mathrm{Wu}^{[55]}$ et al. used polydimethylsiloxane (PDMS) and 2,4-toluene diisocyanate (TDI) to form a PDMS-TDI oligomer as a binding motif to coordinate the cross-linking of other bonds. In PDMS-TDI oligomer, the tetra hydrogen bond with $\mathrm{C}=\mathrm{O}$ and $\mathrm{N}-\mathrm{H}$ was formed, which increased the tetra hydrogen bond in polymer network except the metal bonds with stronger bond energy (Figs. 4a, b). The tetra-hydrogen bond is a weak dynamic bond in the polymer network, which can dissipate the strain energy and endow the polymer with high tensile property and excellent notch insensitivity. In addition, the combination of tetra-hydrogen bond and metal coordination bond, which can form a strong molecular network, makes up for the defects of the raw materials, such as the slow repair speed and the deformability, to give the elastomer a remarkable ability to repair itself (Fig. 4c). The properties of self-healing materials have been changed obviously after the introduction to tetrahydrogen bond. The self-healing elastomer can bear $2.6 \mathrm{MPa}$ high tensile stress, $14.7 \mathrm{MJ} \cdot \mathrm{m}^{-3}$ high toughness, $1700 \%$ stress-deformation and $90 \%$ selfhealing. Due to its preeminent toughness, deformability and electrical signal conduction ability, this kind of self-healing material with advantageous self-healing performance will be 


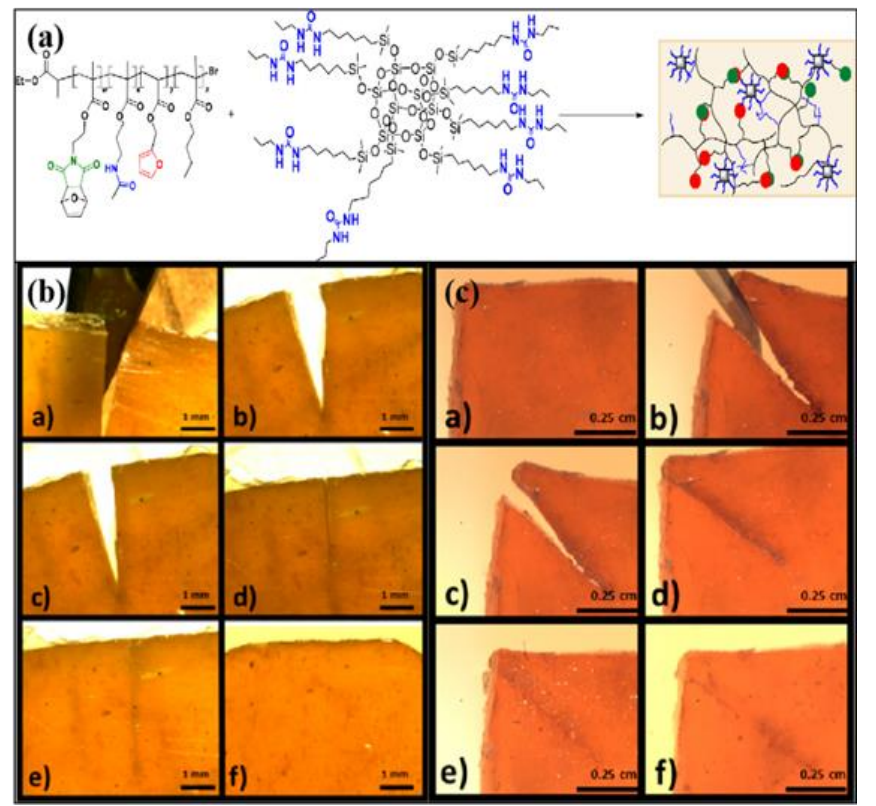

Fig. 5 (a) Structure diagram and molecular chain diagram of the self-healing material. (b) Self-healing condition before improvement. (c) Self-healing condition after improvement, reproduced with the permission from [58], Copyright 2018 American Chemical Society.

used in the fields of electronic skin and intelligent sensor. Soft materials with the ability to deform easily and reversibly hold great promised for applications in broad fields, from wearable electronics and soft robotics to medical devices, automotive engineering, and aerospace exploration. ${ }^{[56]}$

For instance, Zhang ${ }^{[57]}$ et al. used the dynamic crosslinking of hydrogen bonds as the repair mechanism of selfhealing materials, Schäfer ${ }^{[8]}$ et al. were also successful in improving the healing properties of self-healing materials by using hydrogen bonds formed into molecular structures. In the experiment, not only the hydrogen bond was introduced into the molecule, but also the hydrogen bond was used in the molecular network, which could form the hydrogen bond between the macromolecules better, and then improved the self-healing ability of the self-healing material. In the experiment, the polymers were synthesized by the use of 3Acetylaminopropanol, 3-Acetamidopropyl Methacrylate and 2-(4-Butylureido) ethyl Methacrylate, let the amide group or urea group of the three substances as the controlled free radical polymer (CRP), the polymers of these three substances were then subjected to a DA reaction with butyl methacrylate, maleimide, and furan via a CRP, so that the finished product had the function of forming hydrogen bonds in the molecular chain. In the external molecular network, Tetra Ethyl silicate and Tetra Methyl Ammonium Pentahydrate were made into $\mathrm{SiO}_{2}$ octa anion, which was transferred to the $\mathrm{Si}-\mathrm{H}$ terminated cube through conversion, after alkylation of dimethylchlorosilane with Hydrosilylation with $\omega$ bromoalkenes, $\mathrm{NaN}_{3}$ was added to further convert the dimethylchlorosilane into azide-containing cubes, the catalyst was then reduced to an amine and a highly reactive cube was obtained. Ultimately, the cube was transformed into a spherical silicate with hydrogen bond by propyl isocyanate to form an external molecular network with hydrogen bond. Two self-healing mechanisms, DA reaction and hydrogen bond, were used to improve the self-healing ability and mechanical properties of the material. Differential scanning calorimetry (DSC) measurements showed a high peak area between $120{ }^{\circ} \mathrm{C}$ and $200{ }^{\circ} \mathrm{C}$, indicating a significant increase in the number of chains formed into polymers between $120^{\circ} \mathrm{C}$ and $20{ }^{\circ} \mathrm{C}$, it was proved that there are numerous hydrogen bonds between the molecular chain network, and the hydrogen bonds had favorable self-healing properties. The optical microscope images showed that when the cutting depth was $1 \mathrm{~mm}$ and the length was at least $5 \mathrm{~mm}$, when the temperature was $120^{\circ} \mathrm{C}$, the material could heal completely in $30 \mathrm{~min}$, and when the temperature was $70{ }^{\circ} \mathrm{C}$, the material could heal completely in 20 h, the material could heal itself completely (Fig. 5).

Hydrogen bonding can not only improve the mechanical properties and repair methods of the original self-healing materials, but also be used as the main repair mechanism of the new self-healing elastomer materials. A new type of selfhealing silicone rubber (HBSR) was synthesized by the reaction of $\alpha, \omega$-aminopropyl-terminated poly(dimethylsiloxane) (A-PDMS) and ethylene carbonate (EC) by Liu ${ }^{[59]}$ et al., which in contrast to the PDMS and Isocyanate reaction. Hydrogen bonds were formed by the connection with $\mathrm{C}=\mathrm{O}$ on the ethyl carbonate with $\mathrm{N}-\mathrm{H}$ on the amino groups of $\alpha, \omega$-aminopropyl polydimethylsiloxane, and the ring-opening of ethyl carbonate was connected with the two ends of $\alpha, \omega-$ aminopropyl polydimethylsiloxane to form hydroxyl groups, to provide the basis for further hydrogen bond formation. Through the observation of the molecular network structure, we found that hydrogen bonds constituted the self-healing polymer molecular network. These included single hydrogen bonds between hydrogen atoms and hydrogen atoms, and hydrogen bonds between $\mathrm{C}=\mathrm{O}$ and $\mathrm{N}-\mathrm{H}$ and hydroxyl groups in polymers. The multiple forms of hydrogen bonds in polymer networks to provide stable molecular networks and high-efficient and rapid self-healing properties for self-healing materials. On account of the A-PDMS in the raw material had many amino groups, the amino groups could form numerous hydrogen bonds in the molecular chain of the self-healing elastomer, so the cross-linking density of the hydrogen bonds in the material was increased, and the material had better mechanical tensile properties, the tensile strength of the selfhealing material was tested, and the pressure could be up to $0.52 \mathrm{MPa}$. In addition, due to the thermal reversibility of the hydrogen bond, the hydrogen bond of the cross-linked silicone rubber could repair itself under the heating condition. The specimens were put into the oven at $80{ }^{\circ} \mathrm{C}$ for $24 \mathrm{~h}$ of $500 \mathrm{~g}$ pressures, and the healing efficiency was $88.5 \%$, which proved that HBSR had benign cycle healing ability. Polymers that repair themselves after mechanical damage can significantly improve their durability and safety ${ }^{[60]}$ (Fig. 6). Besides, when Hydrogen bond is used as self-healing mechanism by 


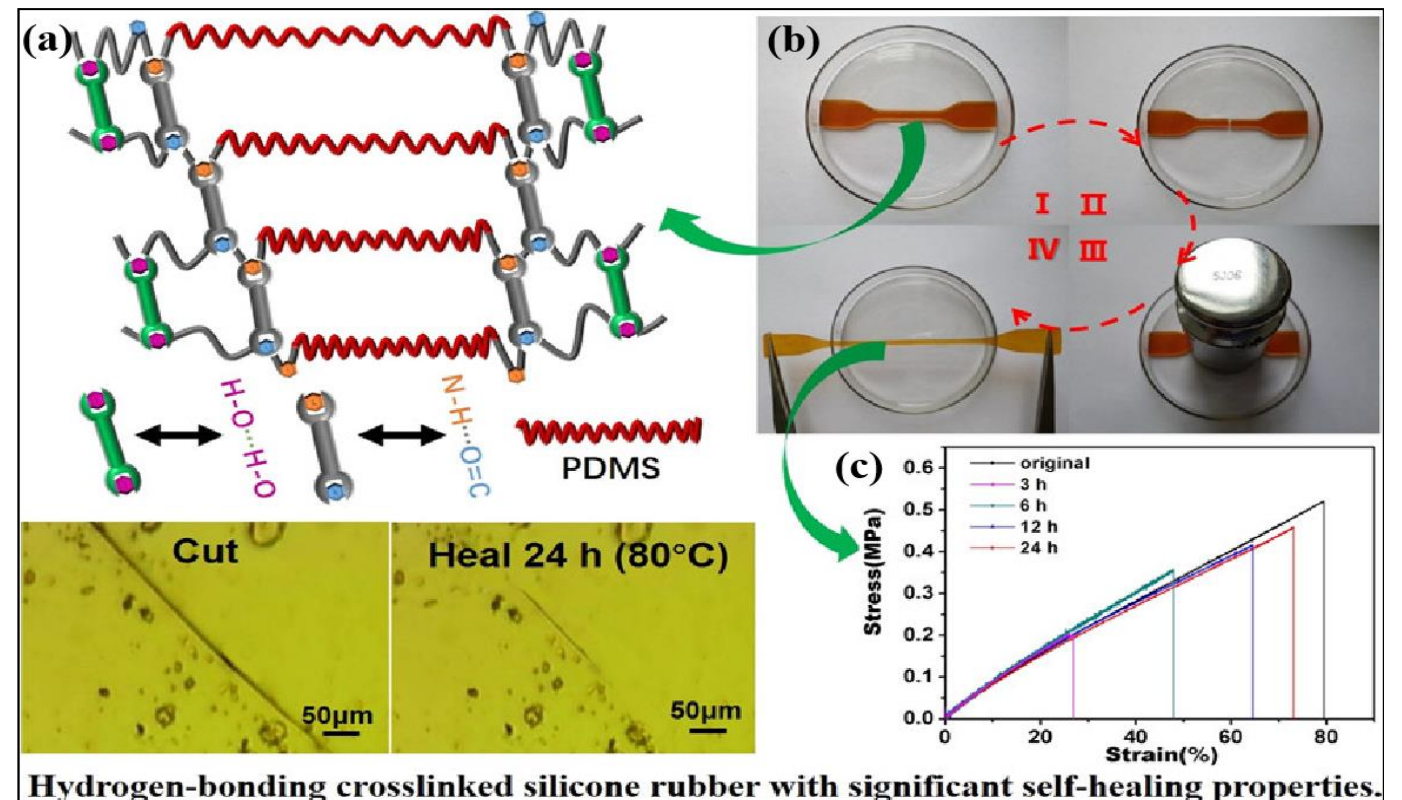

Fig. 6 (a) Molecular structure and self-healing condition of HBSR. (b) HBSR under stress test. (c) Tensile property curve of HBSR, reproduced with the permission from [59], Copyright 2019 American Chemical Society.

Chen $^{[61]}$ et al, it can also improve the repairing ability of selfhealing materials, which type of silicone rubber may be used in soft robots, wearable electronics and scalable circuit structures.

The synthesis of polymeric materials that simultaneously possessed multiple excellent mechanical properties and highefficient self-healability at room temperature is always a huge challenge. ${ }^{[62]}$ As a kind of adjustable bioelastomer, the selfhealing material prepared by hydrogen bonding mechanism has benign self-healing properties and can be easily processed, bio-functionalized and subjected to mechanical dynamic changes, so it can be used in electronic skin. Shin ${ }^{[63]}$ et al. used the hydrogen bond binding ability to make hydrogel electronic skin a seamless interface with strong adhesion during repair. Chen $^{[64]}$ et al. created PSED-U bioelastomers by using new dynamic cross-linking hydrogen bonds, the work used a new synthetic method to produce a modified version of poly(glycerolsebacate) (PGS)-poly (sebacoyl diglyceride) (PSeD) between diglycidyl sebacate and sebacic acid by acid- induced ring-opening polymerization of epoxy groups. PSeD has higher tensile strength $(1.83 \mathrm{MPa})$ and higher maximum elongation at break $(409 \%)$, the self-complementary 2-ureido$4[1 \mathrm{H}]$-pyrimidinones (UPy) can form a quadruple hydrogen bond, synthesis of a new self-healing elastomer PSeD-U with PSeD and UPy. By forming a new dynamic elastomer network, PSeD-U, hydrogen bonds were used to link polymer chains together. Due to the molecular chains of PSeD-U Elastomer cross-linked with each other, this will give PSeD-U thermoplastic and self-healing ability. The experimental results showed that PSeD-U could be completely self-healing within $30 \mathrm{~min}$ at $60{ }^{\circ} \mathrm{C}$, the tensile test showed that the maximum pressure that PSeD-U could bear was $(3.89 \pm 0.65)$ MPa. (Fig. 7). Furthermore, the backbone of PSeD-U had 2urea-4[1h]-pyrimidinone, so it had splendid degradation and biocompatibility. The hydrogen bonds between the UPY MOTIF will connect the polymer chains of PSeD-U to form an elastic 3D network,making PSeD-U as easy to process as Thermoplastic and self-healing.

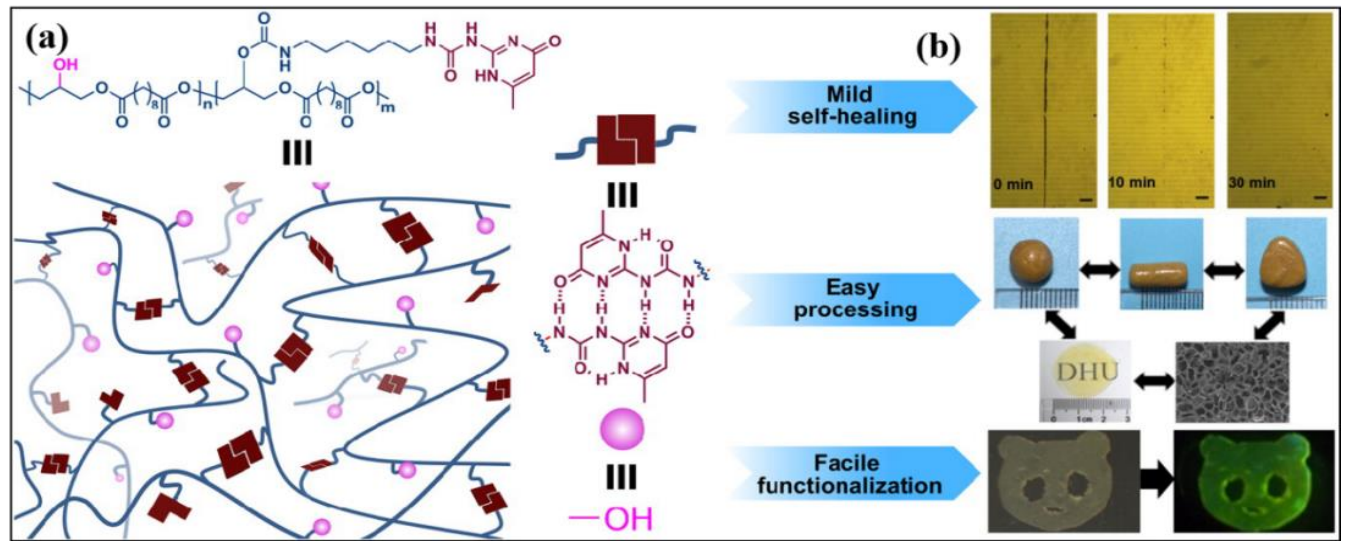

Fig. 7 (a) Structure diagram of PSeD-U.(b) Self-healing condition of PSeD-U, reproduced with the permission from [61], Copyright 2019 American Chemical Society. 
Table 1. Self-repairing materials using hydrogen bond mechanism.

\begin{tabular}{|c|c|c|c|}
\hline Repair method & $\begin{array}{l}\text { Healing } \\
\text { effciency }\end{array}$ & Condition & Ref \\
\hline tetrahydrogen & $90 \%$ & $\begin{array}{l}2.6 \mathrm{MPa} \\
1700 \% \quad \text { stress- } \\
\text { deformation }\end{array}$ & [55] \\
\hline $\begin{array}{l}\text { Hydrogen bonds } \\
\text { molecular chains }\end{array}$ & $100 \%$ & $30 \mathrm{~min}$ at $120^{\circ} \mathrm{C}$ & [58] \\
\hline $\begin{array}{l}\text { Single hydrogen } \\
\text { bond } \\
\text { hydrogen bond }\end{array}$ & $88.5 \%$ & $\begin{array}{l}\text { at } 80^{\circ} \mathrm{C} \\
\text { for } 24 \mathrm{~h}\end{array}$ & [59] \\
\hline $\begin{array}{l}\text { tetrahydrogen } \\
\text { bond }\end{array}$ & $100 \%$ & $\begin{array}{l}30 \text { min at } 60{ }^{\circ} \mathrm{C} \\
\text { Bearing } \quad(3.89 \quad \pm \\
0.65) \mathrm{MPa}\end{array}$ & [64] \\
\hline
\end{tabular}

In summary, hydrogen bonds are widely used in the preparation mechanism of self-healing materials due to their thermal reversibility, easy formation, and high activity, which can also be used as auxiliary bonds to optimize the repair ability and mechanical properties of original self-healing materials (Table 1). The bottleneck problems for their practical applications are long healing time, inferior mechanical strength, and aging sensitivity. ${ }^{[65]}$ Currently, a variety of elastomers with a self-healing capacity and reprocessability have been developed by dynamic chemistry to extend the service life, increase the reliability of polymeric materials, and reduce the waste. ${ }^{[66]}$ Self-healing elastomers made by applying the hydrogen bond mechanism have the characteristics of fast healing speed and excellent mechanical properties, and are widely used in mechanical, electronic and biological fields such as new sensors, electronic skins, and robotic intelligent arms.

\subsection{Self-healing mechanism based on metal bond}

Coordination Bond, also called coordination covalent bond, is a special kind of covalent bond, is formed when the pairs of electrons shared among a covalent bond are supplied by one atom alone and the empty orbitals are provided by the other. When a coordination bond is formed, it is no different from a common covalent bond, except that the two electrons shared between the bonding atoms do not come from one atom, but from the other, and the metal coordination bond is the ligand and metal atoms or ions formed by the coordination bond. ${ }^{[67]}$ Metal Coordination Bond also has the characteristics of covalent bond: directivity and saturation, ${ }^{[68]}$ at the same time, metal coordination bond has certain conductivity ${ }^{[69]}$ to make it more widely used. In recent years, it has been widely used in the field of self-healing to improve the performance of selfhealing materials, so that the materials have better self-healing performance, ${ }^{[70-72]}$ preeminent electrical conductivity. ${ }^{[73]}$ The properties and repair methods of self-healing materials can be improved by introducing metal coordination bonds and combining them with other materials. ${ }^{[74,75]}$

Jing Yang ${ }^{[76]}$ et al. Transformed the material into luminescent self-healing material, because of the dynamic properties of the coordination bond between Ln-L, the tensile properties and self-healing properties of the material are greatly improved. The material can be stretched to 21 times its initial length of a loading rate of $100 \mathrm{~mm}$ Min without cracking, and can lift 2250 without cracking, 6,400 times its own weight, these excellent properties make the material's range of optical and mechanical applications significantly improved. At the same time, Novina Malviya ${ }^{[7]}$ and others had found that lanthanide ions $\left(\mathrm{Ln}^{3+}\right)$ could be used as self-healing materials, and metal coordination bonds were formed between lanthanide ions $\left(\operatorname{Ln}^{3+}\right)$ and the complexes, which make the self-healing materials have unique properties such as luminescence. We had studied the luminescent properties of the material for the following reasons: The unique luminescent properties of lanthanide ions $\left(\mathrm{Ln}^{3+}\right)$, the high coordination number of $\mathrm{Ln}^{3+}$, and the dynamic properties of $\mathrm{Ln}^{3+}$ Ligands.

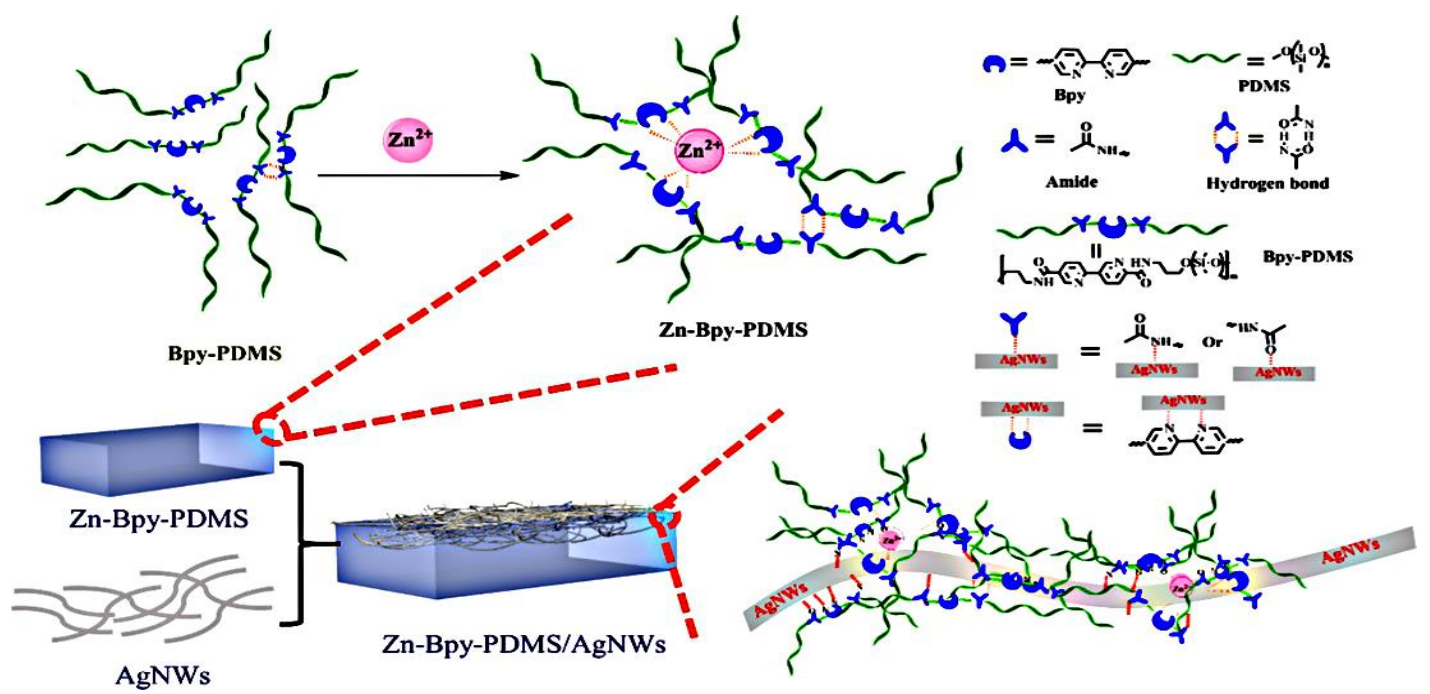

Fig. 8 Schematic diagram of transparent conductor, Zn-BPy-PDMS self-healing polymer. The schematic structure is the interface binding between the self-healing Zn-bpy -PDMS polymer and the Zn-Bpy-PDMS/AgNWs conductor, reproduced with the permission from [78], Copyright 2019 American Chemical Society. 
Guo Ye ${ }^{[78]}$ and others used the dynamic Ag-N bond to enhance the conductivity of the material under the action of $\mathrm{Zn}^{2+}$ Ions, and increased the application range of the material. In the preparation process of self-healing materials, a transparent and stretchable conductor was made, in which a dynamic metal coordination bonds Ag-N bond were formed into the elastic substrates of the conductor, which was used to increase the stability of the substrates. The self-healing elastomer with $\mathrm{AgNW}$ (Ag nanowire) half embedded in PDMS was modified by Bpy Ligand and further cross-linked by $\mathrm{Zn}^{2+}$ Ions (Fig. 8). Although the energy of the dynamic Ag$\mathrm{N}$ bonds formed into AgNW and Bpy was very small, it was enough to make AgNW adapt to deformation and make the material hard. The AgNWs were embedded into the Zn-BpyPDMS polymer, and a dynamic Ag-N bond was introduced into the AgNWs and the Zn-Bpy-PDMS Polymer. It is found that the AgNWs diffusion is promoted by the dynamic Ag-N bond, the resistance of the AgNWs is stabilized under tension, and the AGNWS delamination is prevented by the AgNWs adhesion. The morphology and changes of AgNW on Zn-BpyPDMS and pure PDMS after stretching were studied, it was found that Ag-n bond enhanced the wettability of AgNWs on the surface.

At the same time, the conductor also has preeminent conductivity and transparency, and excellent self-healing performance and low resistance increase.

Lian $\mathrm{Han}^{[79]}$ et al. used the combination and synergy of weak hydrogen bonds and iron coordination bond to enhance the mechanical strength and adhesion of the material, which can be used in health detection, intelligent flexible skin sensors and wearable electronic devices.

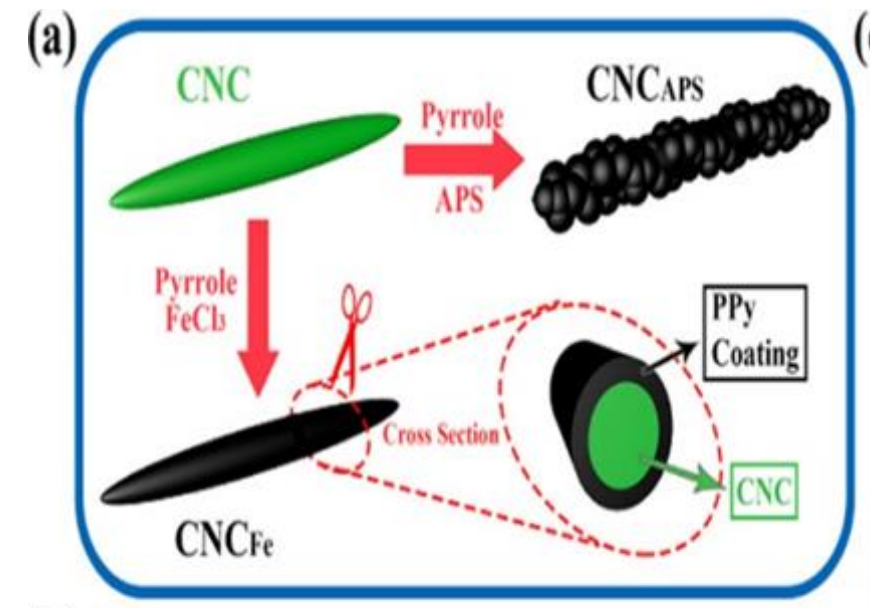

(b)

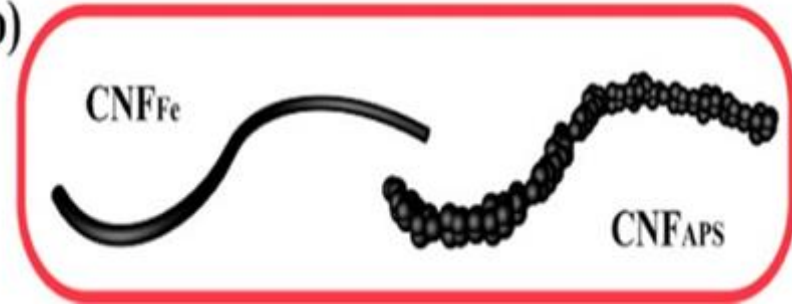

PVA hydrogels are highly elastic, self-healing, adjustable electrical conductivity and strong adhesion to flexible sensor systems; however, they exhibit weak tensile stress due to weak hydrogen bonds. Conductive polymer shells are uniformly coated into $\mathrm{CNC}$ or CNF surfaces to form a strong, selfhealing network with PVA (Fig. 9a). Further chelation of hydrogen bonds with electrically conductive $\mathrm{CNC}$ or $\mathrm{CNF}$ metal ions can further enhance the conductivity and mechanical properties of the composite materials (Fig. 9b), as well as the mechanical strength and self-healing properties of the nano-composite films. At the same time, the system containing $\mathrm{Fe}^{3+}$ Ions can chelate with the polymer, and further improve the mechanical properties and electrical sensitivity (Fig. 9c). This method can stabilize the mechanical strength of the material and enhance the conductivity of PVA/BA hydrogel.

The tensile strength of PVA nanocomposite film is shown as follows (Fig. 10), the tensile strength of pure PVA is only $32.7 \mathrm{MPa}$, while that of CNC/PVA and CNF/PVA is increased to $73.2 \mathrm{MPa}$ and $78.4 \mathrm{MPa}$ respectively. Similarly, CNF APS/PVA and CNF FE/PVA showed the highest tensile strength of $119.5 \mathrm{MPa}$ and $168.0 \mathrm{MPa}$ respectively, compared with PVA and CNF APS/PVA, the strength of CNF FE/PVA nanocomposites increased by $409 \%$ and $40 \%$ respectively. The results show that they have better mechanical strength and flexibility, after repeated cyclic tensile tests, the nanocomposite film has splendid durability. Through the comparison of circuit experiments, we can know that the material has better electrical conductivity after the addition of erric, after self-healing, the material still maintains benign electrical conductivity.

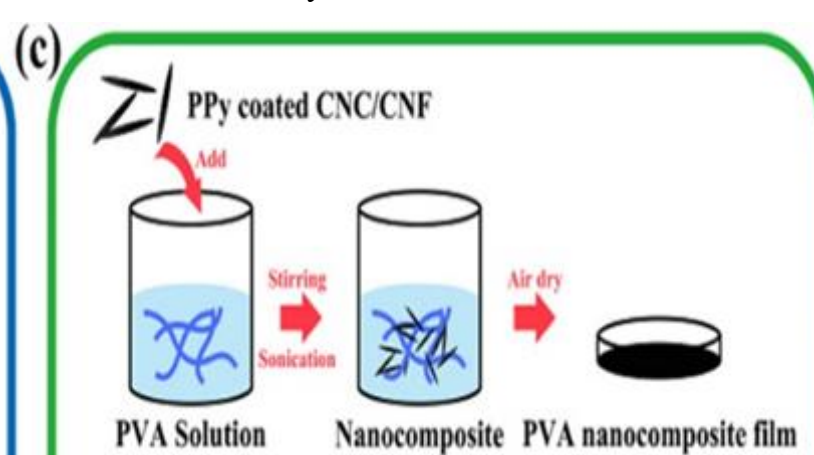

PVA Solution Nanocomposite PVA nanocomposite film

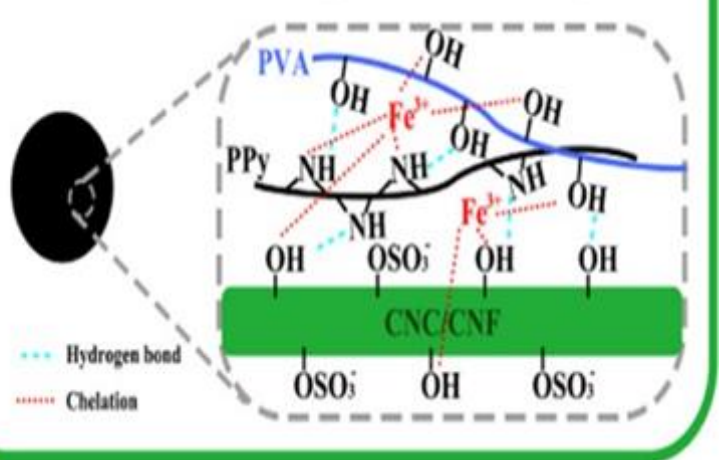

Fig. 9 Synthesis process of PVA nanocomposite film. (a) Synthesis of DIGITALLY controlled CNF-PPy nanocomposites (b) Illustration of CNF-PPy nanocomposites (C) PVA production process with PPy and structure of PVA nanocomposite film, reproduced with the permission from [79], Copyright 2019 American Chemical Society. 
(a)
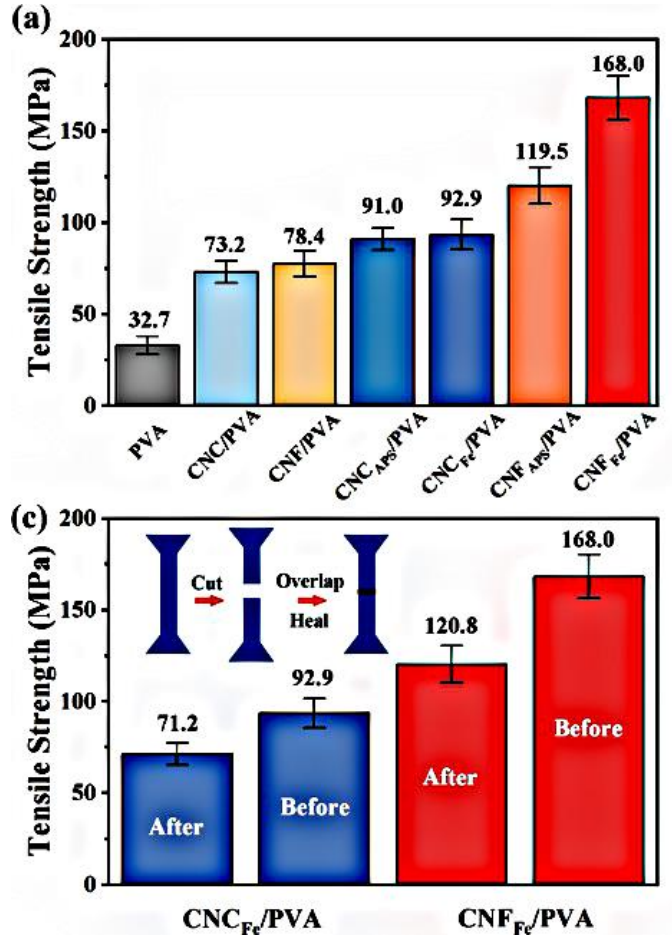

(b)
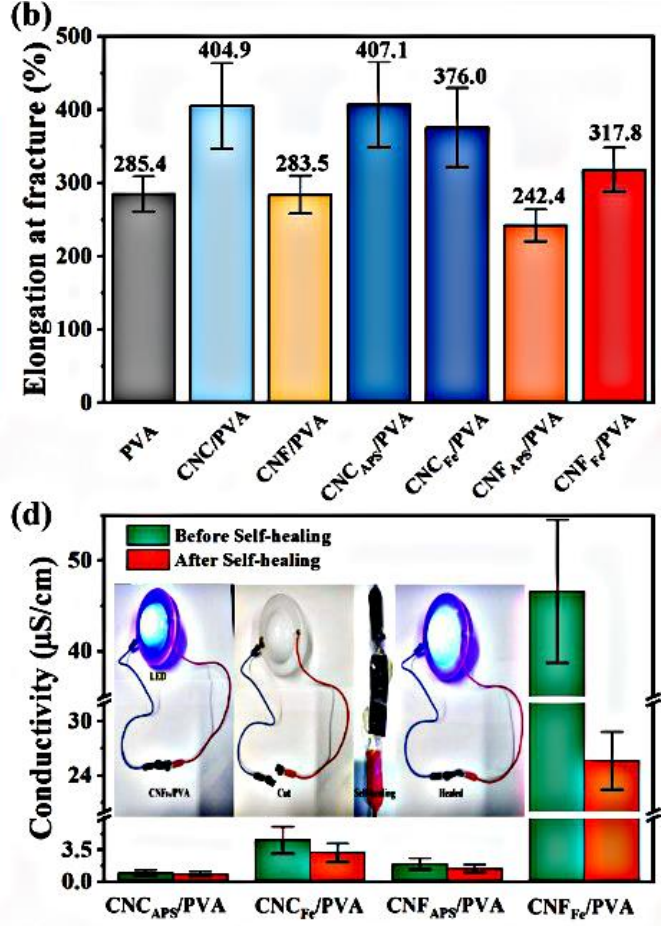

Fig. 10 Performance Test of PVA nanocomposite films (A) Tensile strength of PVA, CNC/PVA, CNF/PVA, CNCAPS/PVA, CNCFe/PVA, CNFAPS/PVA, and CNFFe/PVA. (b) Fracture elongation length of PVA, CNC/PVA, CNF/PVA, CNCAPS/PVA, $\mathrm{CNCFe} / \mathrm{PVA}$, CNFAPS/PVA, and CNFFe/PVA. (c) Changes in tensile strength of CNCFe/PVA and CNFFe/PVA after self-healing. (d) Changes in electrical conductivity of CNCFe/PVA CNCAPS/PVA, CNFFe/PVA and CNFAPS/PVA after self-healing, reproduced with the permission from [79], Copyright 2019 American Chemical Society.

\section{4 Self-healing mechanism based on Diels Alder reaction bonds}

Nowadays, rubbers have been widely used in various fields, including transport vehicles, sports equipment, building materials ${ }^{[80]}$ In research work, we also investigated the use of Diels-Alder reaction bonds ${ }^{[81,82]}$ in the rubber network, a key element of the self-healing concept being the reversible cycloaddition of organic molecules conjugated dienes to substituted alkenes. Chain diffusion, especially based on the mobility of functional groups, is an essential index to evaluate the self-healing ability of Diels-Alder reaction self-healing materials. ${ }^{[83]}$ The nanocomposite material researched by Aishwarya V. Menon ${ }^{[84]}$ has microwave healing ability, reduced graphene oxide $/ \mathrm{Fe}_{3} \mathrm{O}_{4}$ (reduced graphene oxide $/ \mathrm{Fe}_{3} \mathrm{O}_{4}$ FA) and PU (PU-FA), which are mainly furan-bonded, are covalently cross-linked with BMI (bismaleimide) via DA reaction. This material is not only a kind of self-healing material, but also an excellent electromagnetic interference shielding material due to its microwave absorption characteristics. This self-healing ability is triggered by the material absorbing heat generated in the microwave, which is emitted on the material in the form of heat. In order to prove the self-healing ability of these nanocomposites, the samples were pressed for thin films. Moreover, a slit was made in the film and it was exposed to a 900-watt home microwave for 10 min to allow it to heal, After 10 min the sample was removed from the microwave oven and observed under the microscope, the incision was completely healed. Wound healing is due to the reconstitution of the furan and maleimide groups to form Da adducts. Da bonds are much weaker than other types of covalent bonds and may break due to stretching or scratching of these bonds, when a wound is heated by microwave, these bonds are fused and recombined to respond to the heat generated by microwave absorption of the atoms in the sample.

Some of the most elegant routes to achieve intrinsic healing in polymeric materials are Diels-Alder and retroDiels-Alder-based reactions ${ }^{[85]}$ In addition, Da reaction is also considered to be an efficient self-healing cross-linking strategy for the synthesis of solid self-healing hydrogels, ${ }^{[86]}$ and is widely used in tissue engineering. Shao ${ }^{[87]}$ designed a new self-healing hydrogel with cellulose nanocrystals (CNCs) as the reinforcing phase and chemical crosslinking agent through the reversible DA reaction and the furan/maleimide pair. Among these, the furan-maleimide DA adduct is the most prominently studied dynamic covalent bond. ${ }^{[88]}$ The selfhealing nanocomposite hydrogels were prepared by reversible DA reaction (Fig. 11) to polyfunctional cross-linking furan modified CNCs and polymer based polyethylene glycol with maleimide end groups. The results indicated that the mechanical properties and self-healing properties of furan can be modified by adjusting the degree of substitution of furan functional group and the molar ratio of furan to maleimide. What counts were the suspension of such nanoparticles in a cross-linked networks to facilitate the incorporation of polymer matrix in the preparation of hydrogels and enhances the understanding of the relationship between macro- 
functional and micro-structural properties, these results may be useful for the design of reusable and renewable polymer hydrogels.

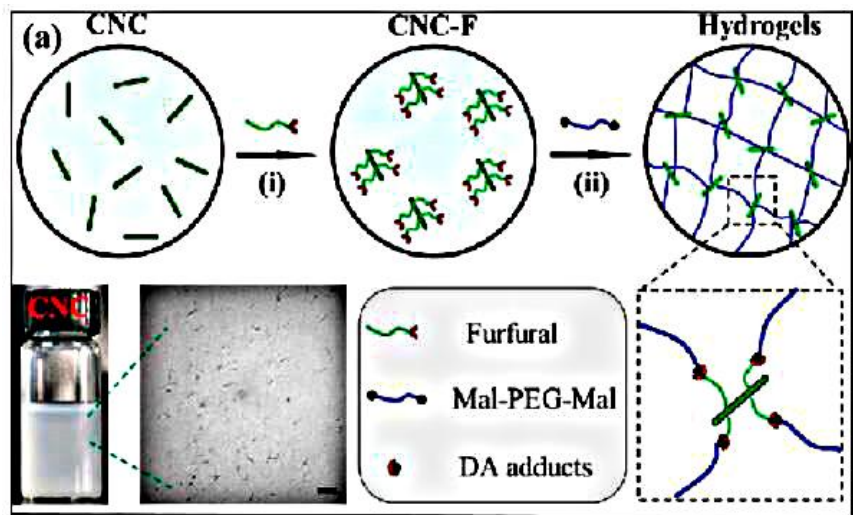

(b)
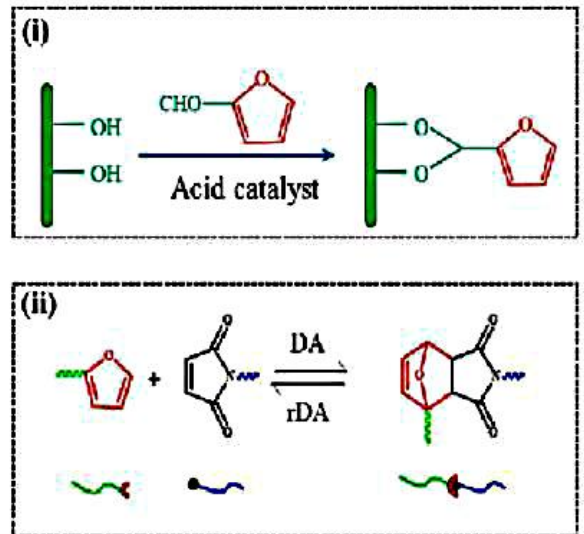

Fig. 11 (a) DA reaction process of $\mathrm{CNC}$ and $\mathrm{CNF}$ molecules under microscope. (b) DA polymerization and DA reversible reactions of composite materials. Reproduced with the permission from [87], Copyright 2017 American Chemical Society.

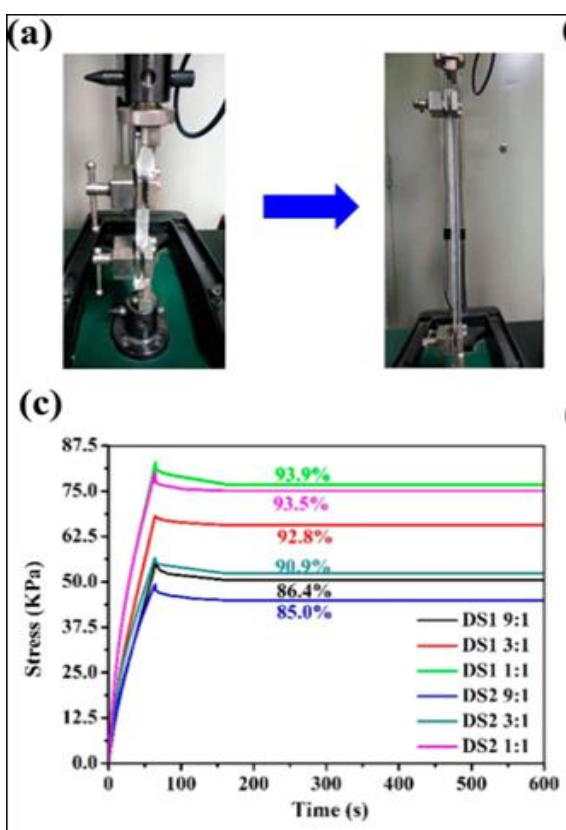

Stress tests were performed on hydrogels of different proportions, which showed that the tensile strength and ultimate durability of the gels enhanced with increasing substitution degree of furyl or decaying mole ratio of furyl to maleimidel. The improvement in mechanical properties is due to the increase of the covalent DA cross-linking point, which helps the gel to bear the stress and improve the failure elongation. At room temperature, the uniaxial tensile test was carried out on the $100 \mathrm{~mm}$ long and $5 \mathrm{~mm}$ diameter rod specimens, and the uniaxial tensile test was carried out at the speed of $60 \mathrm{~mm} / \mathrm{min}$. The unconfined compression test of cylindrical specimens (30 mm high, $20 \mathrm{~mm}$ diameter) was performed at a speed of $10 \mathrm{~mm} / \mathrm{mins}$, to carry out stress relaxation, the specimen was stretched to $200 \%$ (Fig. 12) at the crosshead speed of $60 \mathrm{~mm} / \mathrm{min}$. Afterwards, keeping the strain constant, the time-varying stress relaxation was recorded. The self-recovery properties were investigated by typical tensile test $(60 \mathrm{~mm} / \mathrm{min})$ and compression test $(10 \mathrm{~mm} / \mathrm{min})$, through the tensile test data we can also find DS1 1:1 ratio of selfhealing ability of the strongest, the typical compressive stressstrain curve of the hydrogel is shown in the figure. Similar to the tensile test, the compressive strength of CNC-PEG hydrogels increased with the increase of the degree of substitution of furan and the decrease of the molar ratio of furan to maleimide, which is owing to the increase of stiffness is related to the increase of covalent interaction density of DA bond (Fig. 13e). Through the unconfined compression test of DS1 1:1 hydrogel, which has excellent mechanical properties, strain up to $90 \%$, no fracture or collapse, DA reaction gave the superior thermodynamic property and acid resistance to the hydrogel ${ }^{[89]}$ therefore, it is feasible to realize the self-healing of hydrogel by thermal reversible DA reaction.

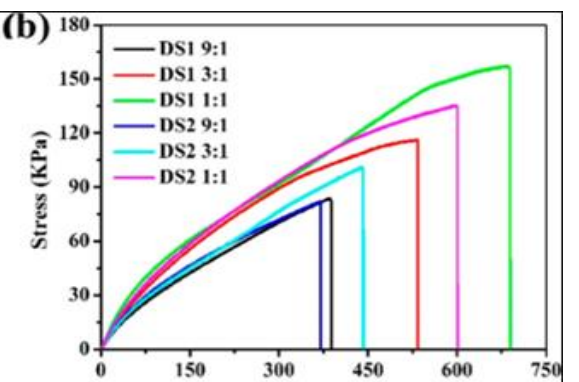

(d)

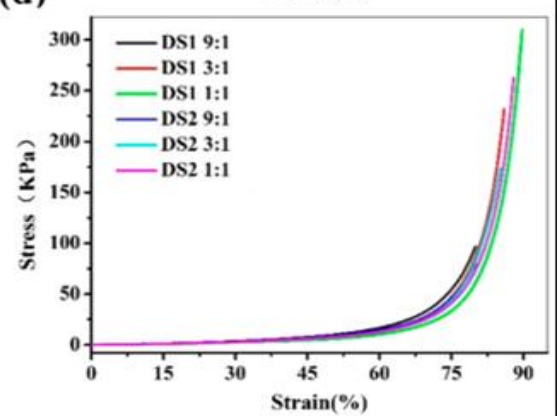

Fig. 12 The mechanical property measurement equipment for the self-healing hydrogel of the product (a), and the mechanical properties measured by uniaxial tensile test (b), unconfined compression test (c), and stress relaxation test (d) respectively, reproduced with the permission form [87], Copyright 2017 American Chemical Society. 


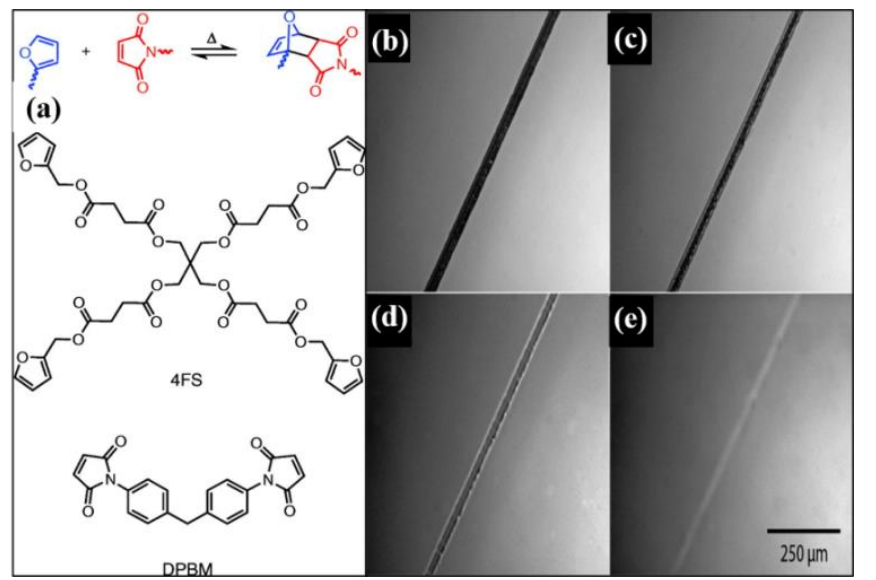

Fig. 13 (a) The molecular structure formula of 4FS and DPBM and the reaction process of DA; (b)(c)(d)(e): In order to repair the surface cracks of the material film, the morphological change is the reaction state corresponding to self-healing, reproduced with the permission from [87], Copyright 2011 American Chemical Society.

Most of the polymers based on reversible covalent bonds are difficult to be remolded at mild condition while the polymers based on noncovalent interaction are usually not stable enough under the conditions of heating or solvents. In order to solve these problems, it is necessary to develop the polymers with the ability of being easily recycled and thermo or solvent stable. ${ }^{[90]}$ The DA reaction can also be applied to the repair mechanism of thermoplastic, ${ }^{[91]}$ the healing of which can be achieved by heating the material and enhancing the polymer diffusion across the interface of the molecular chain. Intrinsic self-healing of polymer mostly operates through reversible inter- and/or intra-molecular interactions and allows healing of the same place in the material for multiple times. ${ }^{[92]}$ When the material is cooled, the structural integrity of the polymer can be restored, and when the DA reaction is used, the molecular chains in the material are heated to form DA cross-linked bonds on the contact surface, and the mechanical properties of the self-healing material after repair are increased. Weziman ${ }^{[93]}$ et al. synthesized tetramic acid by reacting pentaerythritol with succinic anhydride and coupling it with furfuryl alcohol, and replaced the hydrogen on the succinic anhydride with four furan groups to form polymer 4FS. What's more, the 1,10-(methylenedil-4,1-phenylene) bismaleimide (DPBM) was used to react with $4 \mathrm{FS}$, and the maleimide group in DPBM reacted with the furan group in 4FS to form a novel self-healing material (Fig. 13). In the experiment, the apparent peak was observed at $79^{\circ} \mathrm{C}$ due to the large number of chemical bonds formed by the DA polymerization on the contact surface of the material at $79^{\circ} \mathrm{C}$, as measured by the differential scanning calorimetry, this maximizes the peak value, indicating that at $79{ }^{\circ} \mathrm{C}$ the selfhealing material can be rebonded by the use of the DA reaction to form significant chemical bonds with the cracks at the selfhealing material. It can be observed by optical microscope that when the polymer is made into a polymer film, the surface crack at the film can be obviously healed and the mechanical toughness of the self-healing film can be restored after heating for $2 \mathrm{~h}$ of $120^{\circ} \mathrm{C}$, it also shows that self-healing materials can achieve benign self-healing ability through DA reaction.

At the same time, the self-healing materials, which use DA reaction as self-healing mechanism, have been applied in the field of bionic skin (Table 2). Human skin is a unique functional material that perfectly covers body parts having various complicated shapes, spontaneously heals mechanical damage, and senses a touch. ${ }^{\left[{ }^{[4]}\right.}$ Zheng ${ }^{[95]}$ et al. used a polymer with a polyimide structure, DFTPA-PI-MA (2-(4-((4-(bis(3(furan-2-ylmethoxy)-2-hydroxypropyl)amino)phenyl)(p-tolyl) amino)phenyl)-5-((2-methyl-1,3-dioxoisoindolin-5-yl)oxy) isoindoline-1,3-dione-1,1'-(methylenebis(4,1-phenylene))bis (1H-pyrrole-2,5-dione)), to create a self-contained mechanical film that can be used as a biomimetic chameleon skin (Fig. 14). DFTPN(5-((1,3-dioxo-2-(4-(phenyl(p-tolyl)amino)phenyl) isoindolin-5-yl)oxy)-2-methylisoindoline-1,3-dione) was obtained by the reaction to N, N-bis (4-nitrophenyl) benzene14 -diamine with furfuryl glycidyl ether (FGE), that was then reduced and polymerized with 4,4'-oxo-phthalic anhydride (ODPA) to obtain DFTPA-PI, which was then crosslinked with 1,10-(methylene di-4,1-phenyl) maleimide via DA.

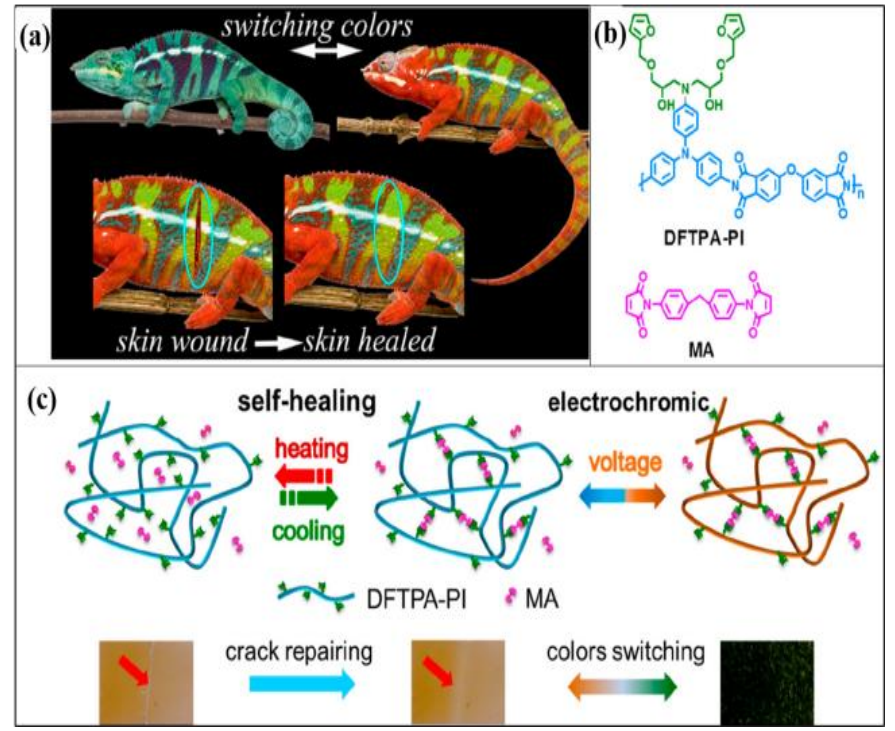

Fig. 14 (a) A biosimulation model of copolymer DFTPA-PI-MA on chameleon as an example.(b) Molecular monomer structure of DFTPA-PI and MA.(c) Structural changes of molecular chain before and after self-healing, reproduced with the permission from [93], Copyright 2018 American Chemical Society.

In order to study the self-healing behavior and process of the copolymer DFTPA-PI-MA, the self-healing process of the copolymer film was recorded at $110^{\circ} \mathrm{C}$, and the self-healing process of the self-healing material was also recorded by electron moving microscope. According to the experiment, the scratch of the copolymer was $20 \mu \mathrm{m}$, and it started to heal in $30 \mathrm{~s}$, and healed in 1 minute, while it took $3 \mathrm{~min}$ for the deeper part of the scratch to heal completely. There was no significant difference in the area of scratch after healing. The copolymer 


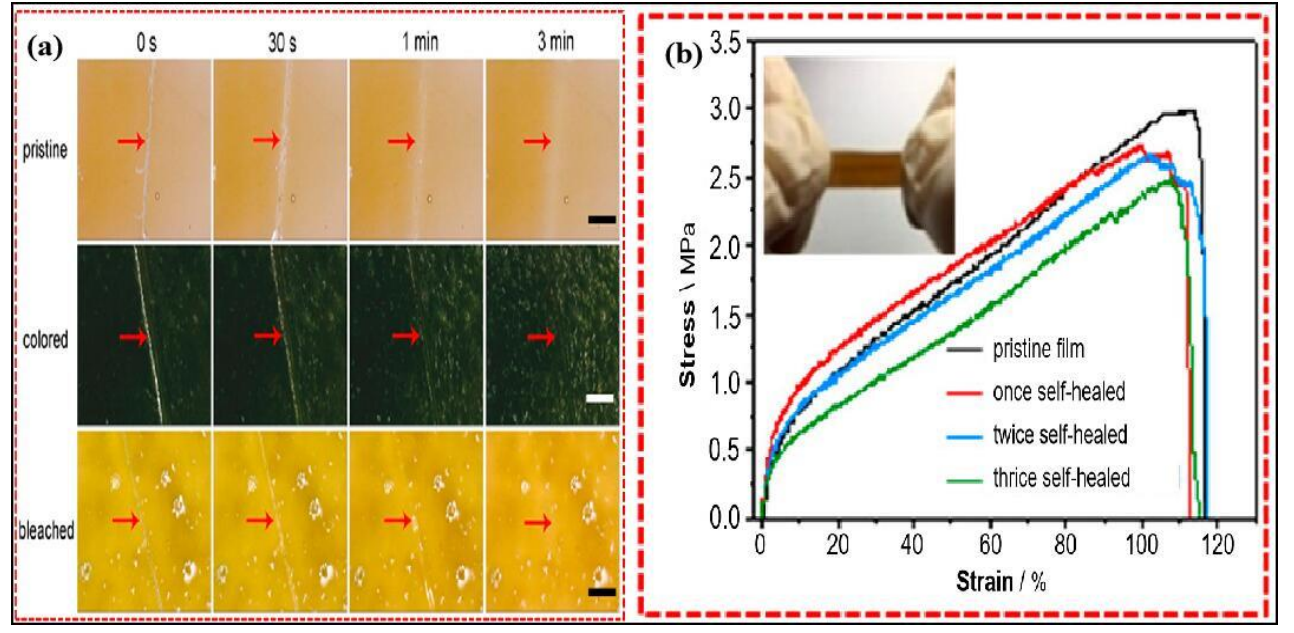

Fig. 15 (a) The change of self-healing phenomena of copolymer DFTPA-PI-MA over time, (b) Test curve of tensile properties of copolymer DFTPA-PI-MA, reproduced with the permission from [93], Copyright 2018 American Chemical Society.

Table 2. Self-repairing materials using DA reaction mechanism

\begin{tabular}{ccc}
\hline Repair methods of self-healing material & Properties of self-healing material & Ref \\
\hline $\begin{array}{c}\text { Graphene oxide/ } \mathrm{Fe}_{3} \mathrm{O}_{4} \mathrm{FA} \text { and } \mathrm{PU}(\mathrm{Pu}-\mathrm{FA}) \\
\begin{array}{c}\text { Modifying } \mathrm{CNCs} \text { with furan and polyethylene } \\
\text { glycol with maleimide terminal group }\end{array}\end{array}$ & Fast DA recombination response & Strong healing ability \\
$\begin{array}{c}\text { The maleimide group in (DPBM) reacts with } \\
\text { furan group in 4FS }\end{array}$ & High healing integrity \\
$\begin{array}{c}\text { The furan group in DFTPA-PI will react with the } \\
\text { MAleimide group in MA }\end{array}$ & Good mechanical ability and deformability & {$[93]$} \\
\hline
\end{tabular}

DFTPA-PI-MA was then split into two halves and was immobilized at $60{ }^{\circ} \mathrm{C}$ for $6 \mathrm{~h}$ to produce DA reaction and fully repaired. After the repair of the material will be tensile test, through the tensile strength experiment to test the mechanical properties of self-healing materials after repair, the tensile strength of the copolymer DFTPA-PI-MA film is $90.7,88.6$ and $86.2 \%$ of that of the raw material (Fig. 15), according to the stress-strain curve, which shows that the copolymer DFTPA-PI-MA film has splendid mechanical properties and deformability. As a chameleon biomimetic material, the copolymer DFTPA-PI-MA also has preeminent colorchanging ability. Flexible ITO (Indium Tin Oxides) (based on PET) and polymer electrolytes (composed of propylene carbonate, PMMA, PEO and $\mathrm{LiClO}_{4}$ ) are combined with the copolymer DFTPA-PI-MA, which can be flexibly changed by voltage. When the voltage was increased from $0 \mathrm{~V}$ to $1.2 \mathrm{~V}$, the copolymer changed from yellow to olive green, the coloring time was about $5 \mathrm{~s}$, and the bleaching time of the original color was about $20 \mathrm{~s}$, the copolymer DFTPA-PI-MA was proved to be an excellent biomimetic material for chameleon skin.

\subsection{Self-healing mechanism based on ionic bonds}

Ionic bonds ${ }^{[96,97]}$ are formed after two or more atoms or chemical groups lose or gain electrons and become ions, scilicet, positive and negative ions between the electrostatic gravity formed by the chemical bond. The ionic bond not only has strong force, but also has the characteristics of nonsaturation and non-direction, in contrast, ionic bonds are nearly as strong as covalent bonds, but stronger than hydrogen bonds.

Ionic bonding is a common method of the design and preparation of self-healing materials, ${ }^{\left[{ }^{[98}\right]}$ such as in the design of self-healing hydrogels, ${ }^{[99-101]}$ the self-healing ability of hydrogels can be obtained by the interaction of iron ions with reversible ions of Gelatin and iron ions with reversible ions of polypyrrole. ${ }^{[102,103]}$ A gelatin-based hydrogel grafted on polypyrrole, which had conductivity, self-healing and injectable properties, was proposed by Wang. ${ }^{[104]}$ By grafting methacrylic anhydrite onto gelatin, they produced doublebond-functional gelatin (GelMA), the common conductive polymer polypyrrole was then grafted onto gelatin (PPyGelMA) by double bond reaction (Figs. 16a and b). Ultimately, polypyrrole grafted gelatin was mixed with Ferric to form a hydrogel (PPy-GelMA-Fe) (Fig. 16c).

Results from Wang et al. showed that the hydrogels had preeminent conductivity due to the addition of polypyrrole and ferric, the self-healing ability of hydrogels was on account of the reversible interaction of iron ions with gelatin and polypyrrole. It was found that the self-healing ability of the hydrogel made it have splendid injectable properties.

Wang ${ }^{[104]}$ compared the self-healing ability of three hydrogel materials with different component contents, used both visuals and qualitative methods to test the hydrogel's 
(a)

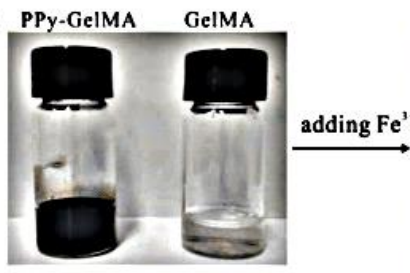

PPy-GelMA-Fe GelMA-Fe

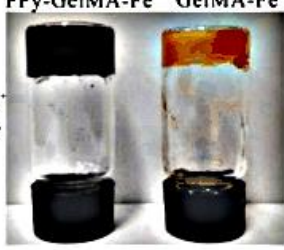

(b)

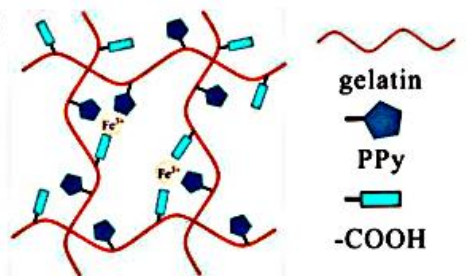

(c)

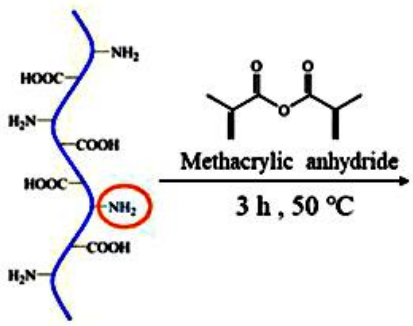

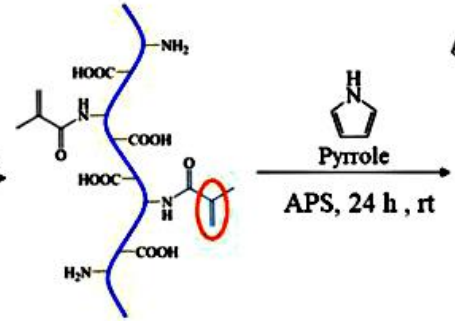

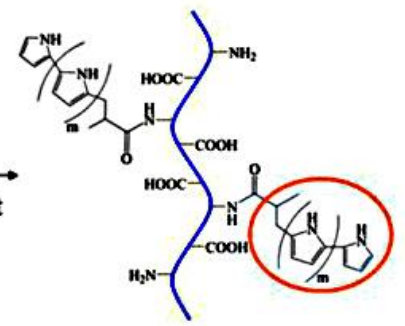

Fig. 16 (a) photos of PPy-GelMA and Gelma and Ferric to form PPy-GelMA hydrogel. (b) schematic diagram of the microstructure of the HYDROGEL network. (c) Schematic diagram of the synthetic route of MA and PPy-GelMA. Reproduced with the permission from [104], Copyright 2020 American Chemical Society.

ability to heal itself, such as cutting and healing of PPyGelMA3-Fe. The results showed that PPy-GelMA3-Fe was able to self-heal in $30 \mathrm{~min}$ at $37^{\circ} \mathrm{C}$, and the compressive strength of the hydrogel can be restored to $70 \%$ after $30 \mathrm{~min}$ of self-healing, which indicated its self-healing ability. It was also found that PPy-GelMA3-Fe, which was cut and then selfhealed, healed faster than the others, and that PPy-GelMA3Fe exhibited higher compressive strength than PPYGELMA2-FE and PPy-GelMA3-Fe (Fig. 17). This is because
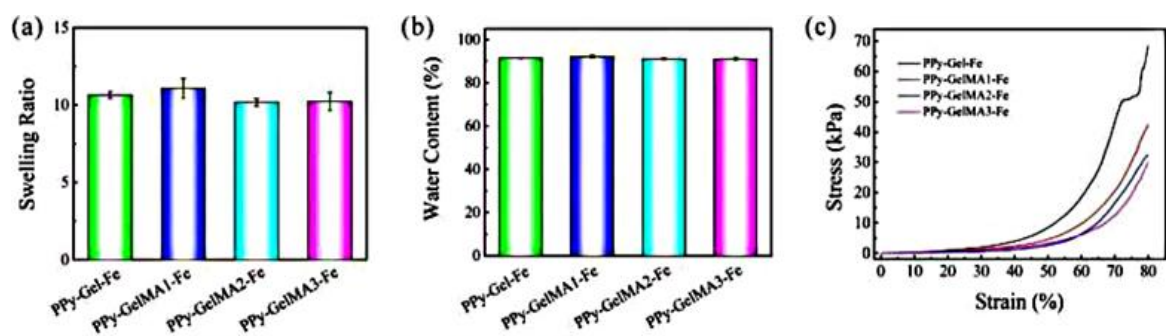

(d) $\left(d_{1}\right)$

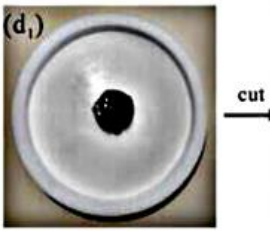

$\left(d_{2}\right)$
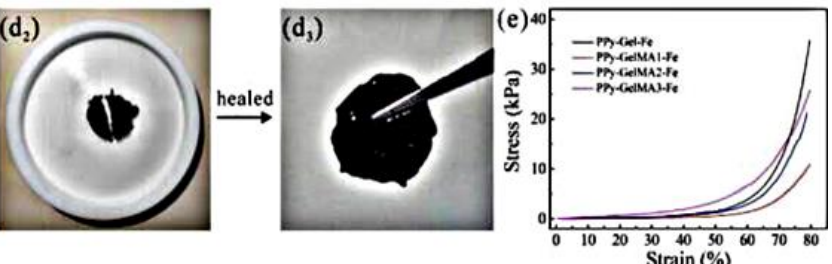

(f)
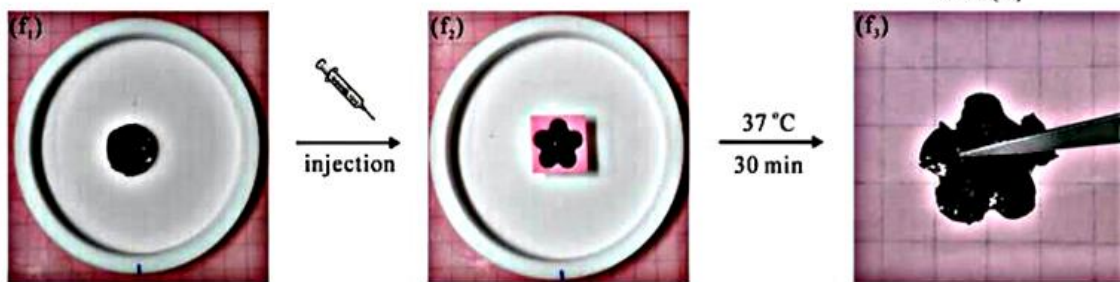

Fig. 17 (a) Swelling ratios (b) water content of the hydrogels in the equilibrium state in PBS at $37{ }^{\circ} \mathrm{C}$ and (c) Schematic diagram of the synthetic route of MA and PPy-GelMA. (d)Cutting and self-healing of PPy-GelMA3-Fe and (e) Compression-rebonding hydrogel stress curve (f) (f1) Photo of PPy-GelMA3-Fe hydrogel (f2) Inject PPy- GelMA3-Fe hydrogel into the flower-shaped mold, (f3) 30 min after self-healing of PPy-GelMA3-Fe hydrogel at $37^{\circ} \mathrm{C}$, reproduced with the permission from [104], Copyright 2020 American Chemical Society. 
(a)

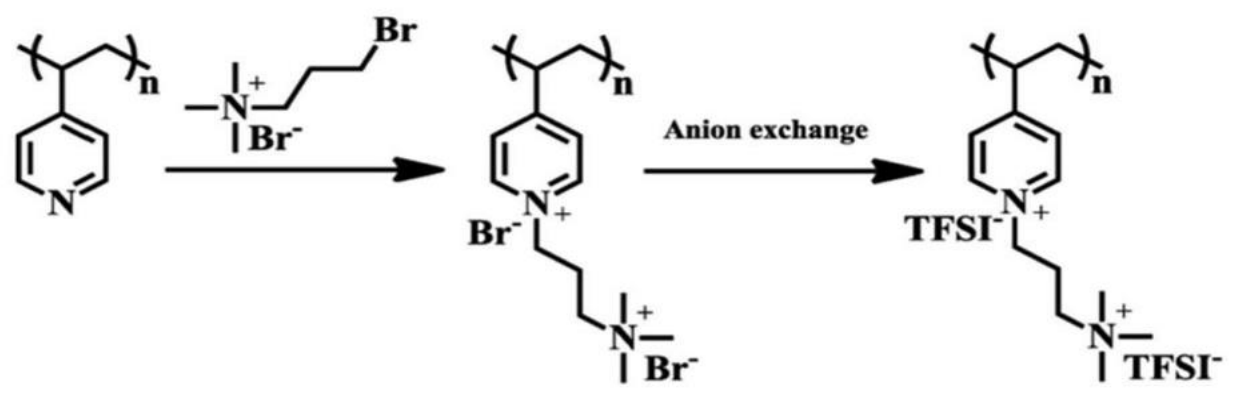

Poly[VPd]

Poly[VPd][TMPN][Br]

Poly[VPd][TMPN][TFSI]

(b)

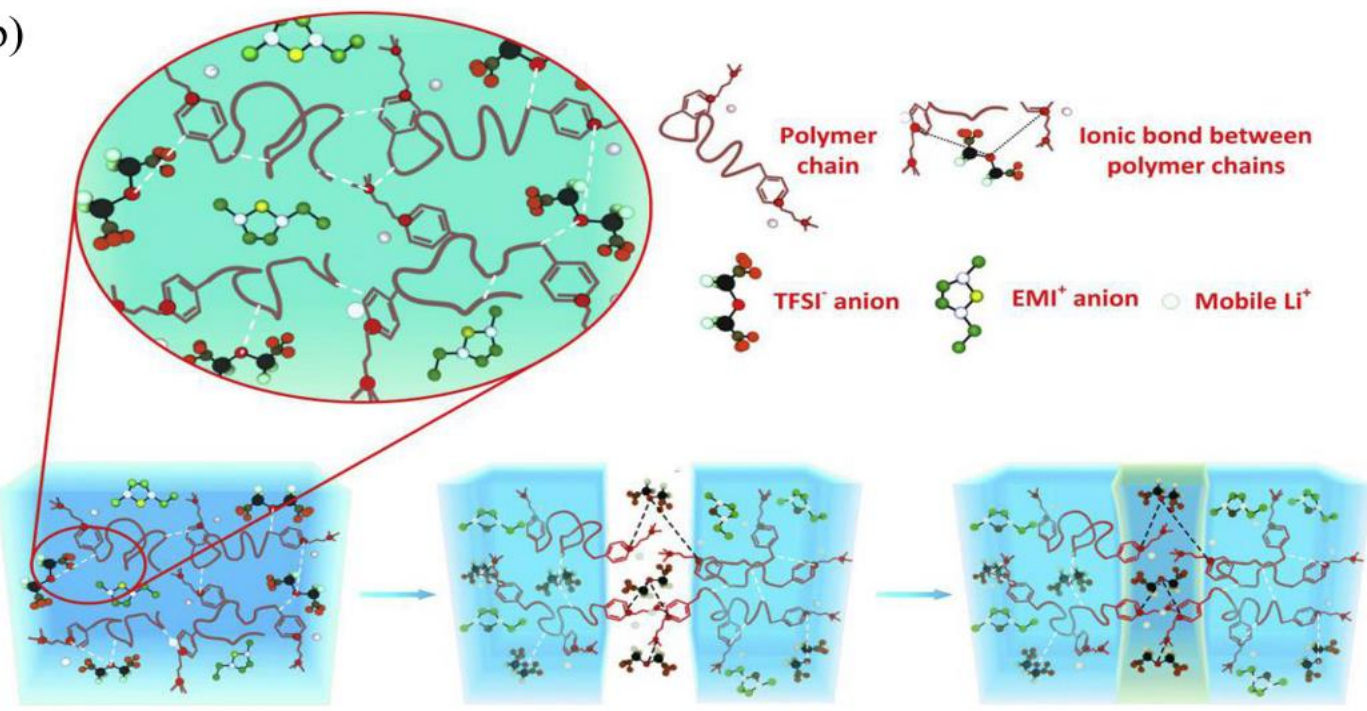

Fig. 18 (a) Schematic diagram of self-healing electrolyte synthesis. (b) An illustration of the mechanism of ionic bond reconstitution during electrolyte self-healing, reproduced with the permission from [109], Copyright 2020 Elsevier.

twisting and bending, which will produce micro- cracks and fracture, which will lead to equipment damage, waste and loss. Therefore, the flexible solid-state lithium battery ${ }^{[108]}$ of selfhealing electrolyte becomes a new breakthrough to solve this problem. Nevertheless, self-healing electrolytes used in lithium batteries have not been widely used due to their low ionic conductivity and limited self-healing ability.

In the latest research, $\operatorname{Tian}^{[109]}$ et al. designed a novel ionic polymer ionic liquid (PILs), poly (4-vinylpyridine) (propyltrimethylammonium) (PVT) as a self-healing electrolyte backbone. In the electrolyte, the gaps between the PVT segments were filled and a composite electrolyte was formed with 1-ethyl-3-methylimidazolium bis (trifluoromethanesulfonyl) imide (EMIMTFSI), ionic liquid (IL) and lithium salt ${ }^{[110]}$ (LiTFSI) form composite electrolytes (Fig. 18a). The sea-island system that interweaves $\mathrm{Li}^{+}$channels and improves the ionic conductivity of the electrolyte ions were formed by a polymer backbone with ionic liquids. Eventually, the composite electrolyte exhibits high self-healing performance, outstanding flexibility and excellent ionic conductivity (Fig. 18b).

Based on this design, it is known that the increased ionic density contributed by the amphoteric polymer chain is vital for the enhancement of the interaction between a large number of cations and anions present in the polymer chain segment and ionic liquid phase.

$\operatorname{Tian}^{[109]}$ then experimented with a certain amount of EMIMTFSI ionic liquid (molecular structure as shown in b) and LiTFSI salt. PVT-acetone solutions can be prepared by dissolving PVT polymers, Litfsi salts and EMIMTFSI ionic liquids in acetone, which was deposited on poly (ethylene terephthalate) (PET). The PVT-acetone sith nitrogen to remove tolution was washed whe acetone solvent, and then the PVTEMIMTFSI composite electrolyte membrane with selfhealing function was formed. In the experiment, Tian et al. used samples of PVT- EMIMTFSI with three different ionic liquid contents. The results showed that the electrochemical stability of PVT- EMIMTFSI electrolyte decreased with the increase of ionic liquid content. From the experimental results, we can know that the PVT- EMIMTFSI composite electrolyte membrane can bend easily and does not break in any way, and has advantageous flexibility and high mechanical strength. When the PVTEMIMTFSI composite electrolyte membrane was cut into two halves directly, the broken composite electrolyte membrane can be restored to its original state without obvious cracks after several minutes of re-contact. 
This is due to in the PVT- EMIMTFSI electrolyte system there are abundant cation and anion interaction ion bond, so the broken solid polymer can be reconstructed by electrostatic force after being reconnected, and the reconstructed ionic bond can recombine the separated polymers, thus, the PVTEMIMTFSI electrolyte has a self-healing function.

In the external cross-linking self-healing materials, ${ }^{[111]}$ it is a common method to introduce microcapsules into the selfhealing materials to realize material repair. Vinita V. Shinde embedded microcapsules containing dicyclopentadiene (DCPD) monomer into the 3D-printed photocurable materials, and confirmed by X-ray nanoCT imaging that the microcapsules were basically evenly distributed in the 3Dprinted photocurable materials. The self-healing mechanism is to restore the mechanical properties and local structural integrity of the surrounding polymer matrix by releasing monomer (DCPD) at the fracture and then conducting catalytic polymerization at room temperature when the material cracks appear. This self-healing mechanism in 3D printed composite structures can repair microcracks in the material and maintain the integrity of the material matrix to prevent or delay the mechanical failure of SLA 3D printed materials. The SENB test showed that the self-healing efficiency could reach $73 \%$ within $72 \mathrm{~h}$.

\section{Conclusions}

Over the years, with the continuous advancement in the field of science and technology, along with the widening of the field of intrinsic self-healing materials ${ }^{[112-117]}$, more and more novel healing systems have emerged, for instance, the realization of rupture and regeneration based on double or multiple hydrogen bonds. Self-healing systems, reversible reactions based on the thermal effect of disulfide bonds to achieve selfhealing systems, and systems that perform chemical reactions based on the energy provided by ultraviolet light to achieve self-healing ${ }^{[18-121]}$, etc. These new healing systems not only have high healed efficiency, but also give polymer materials. Some new properties of the material further widen the application field of the material, nevertheless, there are still some problems with self-healing materials. ${ }^{[122-124]}$

1. The raw material cost of the self-healing material is exorbitant, and the synthesis step and the reaction conditions request are relatively high.

2. At present, the repair condition of self-healing material is too harsh, generally it needs to be heated to a certain temperature or under the condition of electromagnetic radiation to realize the self-healing function, the self-healing function under the condition of normal temperature is irrealizable.

3. The repair speed of self-healing materials should be further improved. Currently, the repair time of self-healing materials usually takes several hours, it is unlikely to happen in the near future with the desire for rapid self-healing. In order to meet the need for the practical application, the repair speed should be improved in the study of self-healing material mechanism.
4. The self-healing performance and other physical properties of self-healing materials will weaken with the increase of using time and repairing times. In order to prolong the using time of self-healing materials and keep the physical properties of self-healing materials, we should continue to study and improve the mechanism and properties of self-healing materials.

5. The physical properties of self-healing materials, such as transparency, flexibility and conductivity, are not perfect, most of them are still limited to theoretical research field, and can not be widely applied in daily life.

6. The use of self-healing materials have yet to be conceived and developed, and needs to be popularized to the general public. In the course of follow-up research, researchers still need to focus on solving the problems mentioned above, in particular, the research should focus on the preparation of materials with superior mechanical properties, fast healing rate and high healing efficiency by selecting cheap monomers, simple synthesis steps and mild reaction conditions, so that it can be widely used in practice. Future self-healing materials will also be used in a wide range of fields, including military equipment, electronics, ${ }^{[125]}$ medical equipment, automobiles, aircraft ${ }^{[126]}$ and building materials, ${ }^{[127]}$ which have bright prospects for use on smartphone and tablet screens, The great significance of which are that it can greatly avoid the waste of resources and funds. At present, once the mobile phone screen is broken, users have to throw it away, which is bound to cause waste, and the application of self-healing materials on the screen will effectively solve this problem. It can be predicted that with the continuous progress of polymer materials design and preparation technology, self-healing materials ${ }^{[128-131]}$ will also play a key role in the broader field.

\section{Acknowledgement}

This work was supported by the Innovation and Entrepreneurship Training Project for Heilongjiang Provincial College Students (S202010225069).

\section{Supporting Information}

Not Applicable.

\section{Conflict of Interest}

There is no conflict of interest.

\section{References}

[1] M. W. Lee, S. Sett, S. S. Yoon, A. L. Yarin, ACS Appl. Mater. Inter., 2016, 8, 18462, doi: 10.1021/acsami.6b05390.

[2] Y. Yang, Y. Kamon, N. A. Lynd, A. Hashidzume, Macromolecules, 2020, 53, 10323, doi:10.1021/acs.Macromol. 0c02080.

[3] Y. Liu, Y. Liu, H. Hu, Z. Liu, X. Pei, B. Yu, P. Yan, F. Zhou, J. Phys. Chem. C, 2015, 119, 7109, doi: 10.1021/jp5120493.

[4] E. Gwak, H. Jeon, E. Song, J. Kim, Nano Lett., 2020, 20, 6706, doi: 10.1021/acs.nanolett.0c02551.

[5] M. Xu, B. Cheng, Y. Sheng, J. Zhou, M. Wang, X. Jiang, X. 
Lu, ACS Appl. Polym. Mater., 2020, 2, 2228, doi: 10.1021/acs apm.0c00154.

[6] X. Xun, Z. Zhang, X. Zhao, B. Zhao, F. Gao, Z. Kang, Q. Liao, Y. Zhang, ACS Nano, 2020, 14, 9066, doi: 10.1021/acsnano.0c04 158.

[7] V. V. Shinde, A. Celestine, L. E. Beckingham, B. S. Beckingham, ACS Appl. Polym. Mater, 2020, 2, 5048, doi: 10.1021/acsapm.0c00872.

[8] M. D. Crall, M. W. Keller, ACS Appl. Mater. Inter., 2017, 9, 6504, doi: 10.1021/acsami.7b00459.

[9] C. Li, Y. Chen, X. Cai, G. Yang, X. S. Sun, ACS Sustain. Chem. Eng., 2020, 8, 3553, doi: 10.1021/acssuschemeng.0c00397.

[10] S. Utrera-Barrios, M. Hernández Santana, R. Verdejo, M.

A. López-Manchado, ACS Omega, 2020, 5, 1902, doi: 10.1021 /acsomega.9b03516.

[11] Y. Qin, J. Wang, C. Qiu, X. Xu, Z. Jin, J. Agr. Food Chem., 2019, 67, 3966, doi: 10.1021/acs.jafc.8b05147.

[12] J. Nie, J. Huang, J. Fan, L. Cao, C. Xu, Y. Chen, ACS Sustain. Chem. Eng., 2020, 8, 13724, doi: 10.1021/acssuschemeng.0c04 136.

[13] J. A. Neal, D. Mozhdehi, Z. Guan, J. Am. Chem. Soc., 2015, 137, 4846, doi: 10.1021/jacs.5b01601.

[14] J. Nie, J. Huang, J. Fan, L. Cao, C. Xu, Y. Chen, ACS Sustain. Chem. Eng., 2020, 8, 13724, doi: 10.1021/acssuschemeng.0c04 136.

[15] Y. Hsu, C. Tai, H. Wu, C. Hou, Y. Liao, W. Liao, G. Haider, Y. Hsiao, C. Lee, S. Chang, Y. Chen, M. Wu, R. Chou, K. P. Bera, Y. Lin, Y. Chen, M. Kataria, S. Lin, C. R. Paul Inbaraj, W. Lin, W. Lee, T. Lin, Y. Lai, Y. Chen, ACS Nano, 2019, 13, 8977, doi: 10.1021/acsnano.9b02858.

[16] J. Yang, Z. Zhang, Y. Yan, S. Liu, Z. Li, Y. Wang, H. Li, ACS Appl. Mater. Inter., 2020, 12, 13239, doi: 10.1021/acsami.9b20 582.

[17] J. Ko, Y. Kim, Y. S. Kim, ACS Appl. Mater. Inter., 2016, 8, 23854, doi: 10.1021/acsami.6b08220.

[18] J. Wang, F. Tang, Y. Wang, Q. Lu, S. Liu, L. Li, ACS Appl. Mater. Inter, 2019, 12, 1558, doi: 10.1021/acsami.9b18646.

[19] Y. Wang, H. Huang, J. Wu, L. Han, Z. Yang, Z. Jiang, R. Wang, Z. Huang, M. Xu, ACS Sustain. Chem. Eng., 2020, 8, 18506, doi: 10.1021/acssuschemeng.0c06258.

[20] R. Zheng, Y. Wang, J. Pan, H. A. Malik, H. Zhang, C. Jia, X. Weng, J. Xie, L. Deng, ACS Appl. Mater. Inter., 2020, 12, 27526 , doi: 10.1021/acsami.0c02337.

[21] Y. Cai, H. Zou, S. Zhou, Y. Chen, M. Liang, ACS Appl. Polym Mater., 2020, 2, 3977, doi: 10.1021/acsapm.0c00638.

[22] M. Xu, B. Cheng, Y. Sheng, J. Zhou, M. Wang, X. Jiang, X. Lu, ACS Appl. Polym. Mater., 2020, 2, 2228, doi: 10.1021/acsapm. 0c00154.

[23] Y. Chen, C. Xia, Z. Shepard, N. Smith, N. Rice, A. M. Peterson, A. Sakulich, ACS Sustain. Chem. Eng., 2017, 5, 3955, doi: 10.1021/acssuschemeng.6b03142.

[24] Y. Wang, C. R. Garcia, Z. Ding, R. Gabrilska, K. P. Rumbaugh, J. Wu, Q. Liu, W. Li, ACS Sustain. Chem. Eng., 2020, 8, 18006, doi: 10.1021/acssuschemeng.0c05730.

[25] S. Das, P. Martin, G. Vasilyev, R. Nandi, N. Amdursky, E.
Zussman, Macromolecules, 2020, 53, 11130, doi: 10.1021/acs. macromol.0c02060.

[26] R. Yang, Y. Yao, Z. Duan, Z. Yuan, H. Tai, Y. Jiang, Y. Zheng, D. Wang, Langmuir, 2020, 36, 3029, doi: 10.1021/acs.langmuir. 0c00221.

[27] H. Xuan, X. Tang, Y. Zhu, J. Ling, Y. Yang, ACS Appl. Bio. Mater, 2020, 3, 1628, doi: 10.1021/acsabm.9b01196.

[28] J. Lei, X. Li, S. Wang, L. Yuan, L. Ge, D. Li, C. Mu, ACS ACS Appl. Polym. Mater., 2019, 1, 1350, doi: 10.1021/acsapm.9b00143.

[29] Q. Zeng, M. S. Desai, H. Jin, J. H. Lee, J Chang, S. Lee, Biomacromolecules, 2016, 17, 2619, doi: 10.1021/acs.biomac.6b00621.

[30] D. Wan, Q. Jiang, Y. Song, J. Pan, T. Qi, G. L. Li, ACS Appl. Polym. Mater., 2019, 2, 879, doi: 10.1021/acsapm.9b01094.

[31] D. Yuan, S. Delpierre, K. Ke, J. Raquez, P. Dubois, I. ManasZloczower, ACS Appl. Mater. Inter., 2019, 11, 17853, doi: 10.10 21/acsami.9b04249.

[32] B. Fan, K. Zhang, Q. Liu, R. Eelkema, ACS Macro. Lett., 2020, 9, 776, doi: 10.1021/acsmacrolett.0c00241.

[33] Y. Wang, C. R. Garcia, Z. Ding, R. Gabrilska, K. P. Rumbaugh, J. Wu, Q. Liu, W. Li, ACS Sustain. Chem. Eng., 2020, 8, 18006, doi: 10.1021/acssuschemeng.0c05730.

[34] X. Wu, J. Wang, J. Huang, S. Yang, ACS Appl. Mater. Inter., 2019, 11, 7387, doi: 10.1021/acsami.8b20303.

[35] H. Gong, Y. Gao, S. Jiang, F. Sun, ACS Appl. Mater. Inter., 2018, 10, 26694, doi: 10.1021/acsami.8b08884.

[36] Y. Shi, Y. Zhang, L. Jia, Q. Zhang, X. Xu, ACS Appl. Mater. Inter., 2018, 10, 36028, doi: 10.1021/acsami.8b13947.

[37] Y. Zhang, M. Li, B. Qin, L. Chen, Y. Liu, X. Zhang, C. Wang, Chem. Mater., 2020, 32, 6310, doi: 10.1021/acs.chemmater.0c0 0096.

[38] T. Li, Z. Xie, J. Xu, Y. Weng, B. Guo, Eur. Polym. J., 2018, 107, 249, doi: 10.1016/j.eurpolymj.2018.08.005.

[39] X. Zheng, H. Yang, Y. Sun, Y. Zhang, Y. Guo, Polymer, 2021, 212, 123111, doi: 10.1016/j.polymer.2020.123111.

[40] W. Li, X. Ni, X. Zhang, Y. Lei, J. Guo, J. Jin, B. You, ACS Appl. Mater. Inter, 2020, 12, 48101, doi: 10.1021/acsami.0c122 66.

[41] Q. Si, Y. Feng, W. Yang, L. Fu, Q. Yan, L. Dong, P. Long, W. Feng, ACS Appl. Mater. Inter., 2018, 10, 29909, doi: 10.1021/ acsami.8b08025.

[42] D. Zhao, Z. Du, S. Liu, Y. Wu, T. Guan, Q. Sun, N. Sun, B. Ren, ACS Appl. Polym. Mater., 2019, 1, 2951, doi: 10.1021/acs apm.9b00656.

[43] H. Wu, X. Liu, D. Sheng, Y. Zhou, S. Xu, H. Xie, X. Tian, Y. Sun, B. Shi, Y. Yang, Polymer, 2021, 214, 123261, doi: 10.1016/ j.polymer.2020.123261.

[44] K. Chang, H. Jia, S. Gu, Eur. Polym. J, 2019, 112, 822, doi: 10.1016/j.eurpolymj.2018.11.005.

[45] M. Liu, J. Zhong, Z. Li, J. Rong, K. Yang, J. Zhou, L. Shen, F. Gao, X. Huang, H. He, Eur. Polym. J., 2020, 124, 109475, doi: 10.1016/j.eurpolymj.2020.109475.

[46] M. Zhang, F. Zhao, Y. Luo, ACS Omega, 2019, 4, 1703, doi: 10.1021/acsomega.8b02923. 
[47] S. S. Rahman, M. Arshad, A. Qureshi, A. Ullah, ACS Appl. Mater. Inter, 2020, 12, 51927, doi: 10.1021/acsami.0c14220.

[48] V. V. Shinde, A. Celestine, L. E. Beckingham, B. S. Beckingham ACS Appl. Polym. Mater, 2020, 2, 5048, doi: 10.10 21/acsapm.0c00872.

[49] X. Li, R. Yu, Y. He, Y. Zhang, X. Yang, X. Zhao, W. Huang, ACS Macro. Lett., 2019, 8, 1511, doi: 10.1021/acsmacrolett.9b 00766.

[50] S. Liu, L. Li, ACS Appl. Mater. Inter., 2017, 9, 26429, doi: 10.1021/acsami.7b07445.

[51] Y. J. Tan, J. Wu, H. Li, B. C. K. Tee, ACS Appl. Mater. Inter. 2018, 10, 15331, doi: 10.1021/acsami.7b19511.

[52] X. Chen, Q. Zhong, C. Cui, L. Ma, S. Liu, Q. Zhang, Y. Wu, L. An, Y. Cheng, S. Ye, X. Chen, Z. Dong, Q. Chen, Y. Zhang, ACS Appl. Mater. Inter., 2020, 12, 30847, doi: 10.1021/acsami. 0c07727.

[53] J. Hou, Z. Chen, S. Zhang, Z. Nie, S. Fan, H. Shu, S. Zhang, B. Li, Y. Cao, Ind. Eng. Chem. Res., 2021, 60, 251, doi: 10.1021/acs.iecr.0c04190.

[54] C. Shao, H. Chang, M. Wang, F. Xu, J. Yang, ACS Appl. Mater. Inter., 2017, 9, 28305, doi: 10.1021/acsami.7b09614.

[55] X. Wu, J. Wang, J. Huang, S. Yang, ACS Appl. Mater. Inter. 2019, 11, 7387, doi: 10.1021/acsami.8b20303.

[56] Y. Zhu, Q. Shen, L. Wei, X. Fu, C. Huang, Y. Zhu, L. Zhao, G. Huang, J. Wu, ACS Appl. Mater. Inter., 2019, 11, 29373, doi: 10.1021/acsami.9b11041.

[57] H. Zhang, D. Wang, N. Wu, C. Li, C. Zhu, N. Zhao, J. Xu, ACS Appl. Mater. Inter., 2020, 12, 9833, doi: 10.1021/acsami.9b22613.

[58] S. Schäfer, G. Kickelbick, Macromolecules, 2018, 51, 6099, doi: 10.1021/acs.macromol.8b00601.

[59] Y. Liu, K. Zhang, J. Sun, J. Yuan, Z. Yang, C. Gao, Y. Wu, Ind. Eng. Chem. Res., 2019, 58, 21452, doi: 10.1021/acs.iecr. 9b03953.

[60] J. A. Neal, D. Mozhdehi, Z. Guan, J. Am. Chem. Soc., 1900, 137, 4846, doi: 10.1021/jacs.5b01601.

[61] X. Chen, Q. Zhong, C. Cui, L. Ma, S. Liu, Q. Zhang, Y. Wu, L. An, Y. Cheng, S. Ye, X. Chen, Z. Dong, Q. Chen, Y. Zhang, ACS Appl. Mater. Inter., 2020, 12, 30847, doi: 10.1021/acsami.0c07727.

[62] Z. Shi, J. Kang, L. Zhang, ACS Appl. Mater. Inter., 2020, 12, 23484, doi: 10.1021 /acsami.0c04414.

[63] S. Shin, S. Kim, H. Jeon, S. Y. Hwang, D. X. Oh, J. Park, ACS Appl. Polym. Mater., 2020, 2, 5352, doi: 10.1021/acsapm.0c00925.

[64] S. Chen, X. Bi, L. Sun, J. Gao, P. Huang, X. Fan, Z. You, Y. Wang, ACS Appl. Mater. Inter, 2016, 8, 20591, doi: 10.1021/acsami.6b05873.

[65] W. Zhao, Y. Liu, Z. Zhang, X. Feng, H. Xu, J. Xu, J. Hu, S. Wang, Y. Wu, S. Yan, ACS Appl. Mater. Inter., 2020, 12, 35445, doi: 10.1021/acsami.0c09045.

[66] C. Fan, Z. Wen, Z. Xu, Y. Xiao, D. Wu, K. Yang, Y. Wang, Macromolecules, 2020, 53, 4284, doi:

10.1021/acs.macromol.0c00239.

[67] J. Joy, D. Danovich, M. Kaupp, S. Shaik, J. Am. Chem. Soc.,
2020, 142, 12277, doi: 10.1021/jacs.0c03957.

[68] R. C. Remsing, M. L. Klein, J. Phys. Chem. B, 2020, 124, 3180, doi: 10.1021/acs.jpcb.0c01798.

[69] Z. Meng, J. Luo, W. Li, K. A. Mirica, J. Am. Chem. Soc., 2020, 142, 21656, doi: 10.1021/jacs.0c07041.

[70] L. Tang, S. Liao, J. Qu, ACS Appl. Mater. Inter, 2018, 10, 27365, doi: 10.1021/acsami.8b09534.

[71] D. E. Fullenkamp, L. He, D. G. Barrett, W. R. Burghardt, P. B. Messersmith, Macromolecules, 2013, 46, 1167, doi: 10.1021 /ma301791n.

[72] Q. Zhang, X. Zhu, C. Li, Y. Cai, X. Jia, Z. Bao, Macromolecules, $\quad 2019, \quad \mathbf{5 2}, \quad 660, \quad$ doi: 10.1021/acs.macromol.8b02414.

[73] J. Yang, T. Wang, R. Guo, D. Yao, W. Guo, S. Liu, Z. Li, Y. Wang, H. Li, ACS Appl. Mater. Inter., 2020, 12, 54026, doi: 10.1021/acsami.0c13509.

[74] X. Chen, Q. Zhong, C. Cui, L. Ma, S. Liu, Q. Zhang, Y. Wu, L. An, Y. Cheng, S. Ye, X. Chen, Z. Dong, Q. Chen, Y. Zhang, ACS Appl. Mater. Inter, 2020, 12, 30847, doi: 10.1021/acsami.0c07727.

[75] S. Han, C. Liu, X. Lin, J. Zheng, J. Wu, C. Liu, ACS Appl. Polym. Mater., 2019, 2, 996, doi: 10.1021/acsapm.9b01198.

[76] J. Yang, Z. Zhang, Y. Yan, S. Liu, Z. Li, Y. Wang, H. Li, ACS Appl. Mater. Inter, 2020, 12, 13239, doi: 10.1021/acsami.9b20582.

[77] N. Malviya, R. Ranjan, C. Sonkar, S. M. Mobin, S. Mukhopadhyay, ACS Appl. Polym. Mater., 2019, 2, 8005, doi: 10.1021/acsanm.9b02064.

[78] G. Ye, Z. Song, T. Yu, Q. Tan, Y. Zhang, T. Chen, C. He, L. Jin, N. Liu, ACS Appl. Mater. Inter, 2019, 12, 1486, doi: 10.1021/acsami.9b17354.

[79] L. Han, S. Cui, H. Yu, M. Song, H. Zhang, N. Grishkewich, C. Huang, D. Kim, K. M. C. Tam, ACS Appl. Mater. Inter., 2019, 11, 44642, doi: 10.1021/acsami.9b17030.

[80] J. Bai, H. Li, Z. Shi, J. Yin, Macromolecules 2015, 48, 3539, doi: 10.1021/acs.macromol.5b00389.

[81] P. A. Pratama, M. Sharifi, A. M. Peterson, G. R. Palmese, ACS Appl. Mater. Inter., 2013, 5, 12425, doi: 10.1021/am403459e. [82] S. Schäfer, G. Kickelbick, ACS Appl. Nano Mater., 2018, 1, 2640, doi: 10.1021/acsanm.8b00308.

[83] P. K. Behera, S. K. Raut, P. Mondal, S. Sarkar, N. K. Singha, ACS Appl. Polym. Mater., 2021, 3, 847, doi: 10.1021/acsapm.0c01179.

[84] A. V. Menon, G. Madras, S. Bose, ACS Omega, 2018, 3, 1137, doi: 10.1021/acsomega.7b01845.

[85] U. Lafont, H. van Zeijl, S. van der Zwaag, ACS Appl. Mater. Inter, 2012, 4, 6280, doi: 10.1021/am301879z.

[86] S. Li, J. Yi, X. Yu, H. Shi, J. Zhu, L. Wang, ACS Biomater. Sci. Eng., 2018, 4, 872, doi: 10.1021/acsbiomaterials.7b00818.

[87] C. Shao, M. Wang, H. Chang, F. Xu, J. Yang, ACS Sustain. Chem. Eng., 2017, 5, 6167, doi: 10.1021/acssuschemeng.7b01060.

[88] T. T. Truong, S. H. Thai, H. T. Nguyen, D. T. T. Phung, L. T. Nguyen, H. Q. Pham, L. T. Nguyen, Chem. Mater, 2019, 31, 2347, doi: 10.1021/acs.chemmater.8b04624. 
[89] S. Li, J. Yi, X. Yu, H. Shi, J. Zhu, L. Wang, ACS Biomater. Sci. Eng., 2018, 4, 872, doi: 10.1021/acsbiomaterials.7b00818.

[90] Z. Feng, B. Yu, J. Hu, H. Zuo, J. Li, H. Sun, N. Ning, M. Tian, L. Zhang, Ind. Eng. Chem. Res., 2018, 58, 1212, doi: 10.1021/acs.iecr.8b05309.

[91] S. Park, B. Shin, S. Jang, K. Chung, ACS Appl. Mater. Inter, 2019, 12, 3953, doi: 10.1021/acsami.9b19272.

[92] L. F. Fan, M. Z. Rong, M. Q. Zhang, X. D. Chen, ACS Appl. Mater. Inter., 2018, 10, 38538, doi: 10.1021/acsami.8b15636.

[93] H. Weizman, C. Nielsen, O. S. Weizman, S. Nemat-Nasser, J. Chem. Educ., 2011, 88, 1137, doi: 10.1021/ed101109f.

[94] S. Park, B. Shin, S. Jang, K. Chung, ACS Appl. Mater. Inter., 2019, 12, 3953, doi: 10.1021/acsami.9b19272.

[95] R. Zheng, Y. Wang, C. Jia, Z. Wan, J. Luo, H. A. Malik, X. Weng, J. Xie, L. Deng, ACS Appl. Mater. Inter., 2018, 10, 35533, doi: $10.1021 /$ acsami.8b13249.

[96] J. Joy, D. Danovich, M. Kaupp, S. Shaik, J. Am. Chem. Soc., 2020, 142, 12277, doi: 10.1021/jacs.0c03957.

[97] D. B. Chesnut, J. Chem. Theory. Comput., 2008, 4, 1637, doi: $10.1021 /$ ct800326n.

[98] F. B. Madsen, L. Yu, A. L. Skov, ACS Macro. Lett., 2016, 5, 1196, doi: 10.1021/acsmacrolett.6b00662.

[99] S. Das, P. Martin, G. Vasilyev, R. Nandi, N. Amdursky, E. Zussman, Macromolecules, 2020, 53, 11130, doi: 10.1021/acs.macromol.0c02060.

[100] S. Yin, J. Liu, D. Wu, S. Chen, W. Xia, ACS Appl. Polym. Mater., 2020, 2, 448, doi: 10.1021/acsapm.9b00905.

[101] Y. Zeng, Y. Li, G. Liu, Y. Wei, Y. Wu, L. Tao, ACS Appl. Polym. Mater., 2019, 2, 404, doi: 10.1021/acsapm.9b00874.

[102] Z. A. Chaleshtari, R. Foudazi, ACS Appl. Polym. Mater., 2020, 2, 3196, doi: 10.1021/acsapm.0c00362.

[103] J. Stejskal, I. Sapurina, J. Vilčáková, M. Jurča, M. Trchová, Z. Kolská, J. Prokeš, I. Křivka, ACS Appl. Polym. Mater., 2021, 3, 1107, doi: 10.1021/acsapm.0c01331.

[104] S. Wang, J. Lei, X. Yi, L. Yuan, L. Ge, D. Li, C. Mu, ACS Appl. Polym. Mater., 2020, 2, 3016, doi: 10.1021/acsapm.0c00468.

[105] A. B. Ihsan, T. L. Sun, T. Kurokawa, S. N. Karobi, T. Nakajima, T. Nonoyama, C. K. Roy, F. Luo, J P. Gong, Macromolecules, 2016, 49, 4245, doi: 10.1021/acs.macromol.6b00437.

[106] Y. Wang, H. Huang, J. Wu, L. Han, Z. Yang, Z. Jiang, R. Wang, Z. Huang, M. Xu, ACS Sustain. Chem. Eng., 2020, 8, 18506, doi: 10.1021/acssuschemeng.0c06258.

[107] C. Cui, Q. Fu, L. Meng, S. Hao, R. Dai, J. Yang, ACS Appl. Bio. Mater., 2020, 4, 85-121, doi: 10.1021/acsabm.0c00807.

[108] Y. H. Jo, S. Li, C. Zuo, Y. Zhang, H. Gan, S. Li, L. Yu, D. He, X. Xie, Z. Xue, Macromolecules, 2020, 53, 1024, doi: 10.1021/acs.macromol.9b02305.

[109] X. Tian, P. Yang, Y. Yi, P. Liu, T. Wang, C. Shu, L. Qu, W. Tang, Y. Zhang, M. Li, B. Yang, J Power. Sources, 2020, 450, 227629, doi: 10.1016/j.jpowsour.2019.227629.

[110] D. Li, L. Yuan, G. Liang, A. Gu, Ind. Eng. Chem. Res., 2020, 59, 6600, doi: 10.1021/acs.iecr.9b06977.

[111] V. V. Shinde, A. Celestine, L. E. Beckingham, B. S.
Beckingham, ACS Appl. Polym. Mater, 2020, 2, 5048, doi: 10.1021/acsapm.0c00872.

[112] X. Fang, Z. Pan, A. Chen, Constr. Build. Mater., 2021, 274, 121999, doi: 10.1016/j.conbuildmat.2020.121999.

[113] M. Mohamadhoseini, Z. Mohamadnia, Coordin. Chem.

Rev., 2021, 432, 213711, doi: 10.1016/j.ccr.2020.213711.

[114] Q. Fu, Q. Yan, L. Lv, H. Fu, React. Funct. Polym., 2020, 157, 104744, doi: 10.1016/j.reactfunctpolym.2020.104744.

[115] M. Zhu, J. Liu, L. Gan, M. Long, Eur. Polym. J., 2020, 129, 109651, doi: 10.1016/j.eurpolymj.2020.109651.

[116] K. Ning, B. Loomans, C. Yeung, J. Li, F. Yang, S. Leeuwenburgh, Dent. Mater, 2021, 37, 403, doi: 10.1016/j.dental. 2020.11 .025 .

[117] H. Kim, H. M. Son, J. Seo, H. K. Lee, Constr. Build. Mater. 2021, 274, 122094, doi: 10.1016/j.conbuildmat.2020.122094.

[118] D. Zhao, S. Liu, Y. Wu, T. Guan, N. Sun, B. Ren, Prog. Org. Coat., 2019, 133, 289, doi: 10.1016/j.porgcoat.2019.04.060.

[119] P. Li, W. Guo, Z. Lu, J. Tian, X. Li, H. Wang, Prog. Org. Coat., 2021, 151, 106046, doi: 10.1016/j.porgcoat.2020.106046. [120] H. Liu, B. Xu, X. Yang, Z. Li, Z. Mo, Y. Yao, S. Lin, Compos. Commun., 2020, 19, 233, doi: 10.1016/j.coco.2020.03.014.

[121] Y. Cheng, X. Xiao, K. Pan, H. Pang, Chem. Eng. J., 2020, 380, 122565, doi: 10.1016/j.cej.2019.122565.

[122] S. Castanié, T. Carlier, F. O. Méar, S. Saitzek, J. Blach, R. Podor, L. Montagne, ACS Appl. Mater. Inter., 2015, 8, 4208, doi: 10.1021/acsami.5b12049.

[123] Y. Cai, H. Zou, S. Zhou, Y. Chen, M. Liang, ACS Appl. Polym. Mater., 2020, 2, 3977, doi: 10.1021/acsapm.0c00638.

[124] J. Sun, J. Wang, M. Chen, X. Pu, G. Wang, L. Li, G. Chen, Y. Cai, X. Gu, B. Z. Tang, Chem. Mater, 2019, 31, 5683, doi: 10.1021/acs.chemmater.9b01611.

[125] S. Wu, J. Li, G. Zhang, Y. Yao, G. Li, R. Sun, C. Wong, ACS Appl. Mater. Inter., 2017, 9, 3040, doi: 10.1021/acsami.6b15476.

[126] W. Guo, Y. Jia, K. Tian, Z. Xu, J. Jiao, R. Li, Y. Wu, L. Cao, H. Wang, ACS Appl. Mater. Inter, 2016, 8, 21046, doi: 10.1021/acsami.6b06091.

[127] B. Pang, J. Qian, Y. Zhang, Y. Jia, H. Ni, S. D. Pang, G. Liu, R. Qian, W. She, L. Yang, Z. Liu, ACS Appl. Mater. Inter., 2019, 11, 29242, doi: 10.1021/acsami.9b08303.

[128] J. Chen, Y. Huang, X. Ma, Y. Lei, Adv. Compos. Hybrid Mater., 2018, 1, 94, doi: 10.1007/s42114-017-0009-y.

[129] S. Kordjazi, K. Kamyab, N. Hemmatinejad, Adv. Compos. Hybrid Mater., 2020, 3, 167, doi: 10.1007/s42114-020-00150-8.

[130] F. Liu, J. Kong, C. Luo, F. Ye, X. Luan, N. Tian, Y. Liu, H. Zhang, J. Gu, Y. Tang, Adv. Compos. Hybrid Mater., 2018, 1, 506, doi: 10.1007/s42114-018-0044-3.

[131] C. Liu, Q. Yin, X. Li, L. Hao, W. Zhang, Y. Bao, J. Ma, Adv. Compos. Mater., 2021, 4, 138, doi: 10.1007/s42114-021-002063.

[132] J. Guo, X. Li, H. Liu, D. P. Young, G. Song, K. Song, J. Zhu, J. Kong, Z. Guo, Adv. Compos. Mater., 2021, 4, 51, doi: 10.1007/s42114-021-00211-6.

[133] J. Guo, Z. Chen, W. Abdul, J. Kong, M. A. Khan, D. P. Young, J. Zhu, Z. Guo, Adv. Compos. Mater, 2021, doi: 
10.1007/s42114-021-00242-Z.

\section{Authors Information}

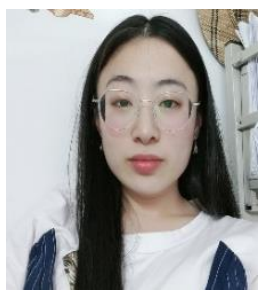

Tingting Song is a junior student currently studying in the School of Chemistry, Chemical Engineering and Resource Utilization, Northeast Forestry University, China. Her research topic is the application of self-healing materials in $3 D$ printing.

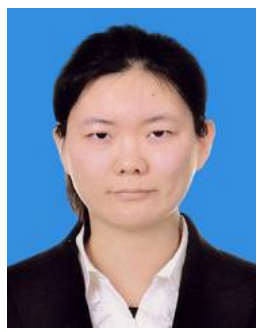

Zhuohan Ji is currently studying chemical engineering and technology at Northeast Forestry University, China. Her current research focuses on application of selfhealing in electronic skin.

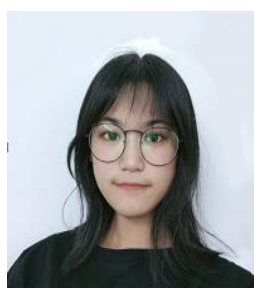

Hongling Zhou is now studying in the third year of junior year at the School of Chemistry, Chemical Engineering and Resource Utilization, Northeast Forestry University, China. Her current research focuses on self-healing materials.

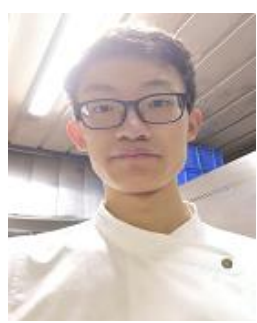

Bojun Jiang is studying for her bachelor's degree in the School of Chemistry, Chemical Engineering and Resource Utilization at Northeast Forestry University, China. His research focuses on the application of cellulosic gels in medical sensing.

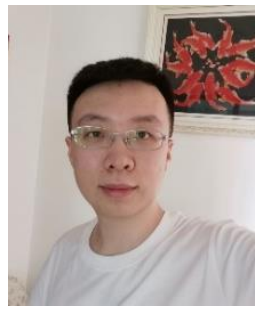

Yida Li a junior student in chemical engineering and technology from Northeast Forestry University. His main research area is the synthesis of selfhealing materials. He has studied organic chemistry and materials synthesis.

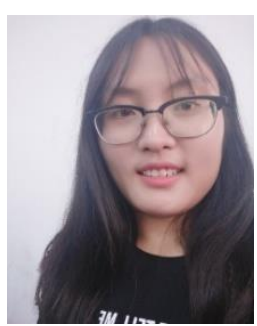

Nan Wen received her $B . S c$. degree from Heilongjiang University of Science and Technology, China. She is now pursing the M.Sc. degree at the College of Chemistry, Chemical Engineering and Resource Utilization at Northeast Forestry University, China. Her research focuses on flexible sensor and conductive

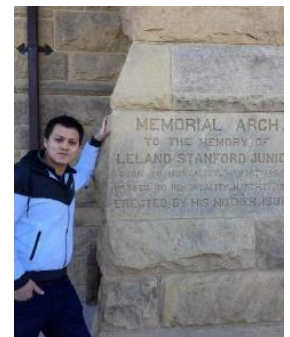

Dawei Jiang is currently an associate Professor in College of Chemistry, Chemical Engineering and Resource Utilization at Northeast Forestry University, China. He received his $P h D$ degree from Harbin Institute of Technology, China. He once studied at Lamar University, Texas, USA. His research interests include advanced self-healing materials, flexible sensor and nanomaterials.

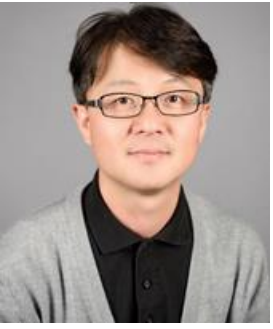

Ilwoo Seok is an associate professor of Mechanical Engineering at Arkansas State University, USA. He received his Ph.D. from the University of California, Los Angeles. Seok explores advanced materials science and micro/nanomanufacturing research and applies energy, environments, and bio-medicals.

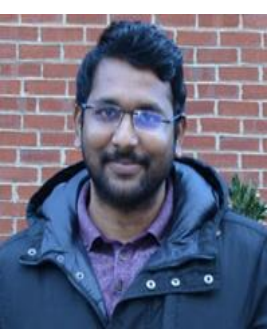

Vignesh Murugadoss obtained his Ph.D. degree from the Centre for Nanoscience and Technology at Pondicherry University, Puducherry. He carried out the Indo-US BASE internship at Integrated Composites Laboratory, Department of Chemical and Biomolecular Engineering at the University of Tennessee, Knoxville, USA. His research interests focus on advanced functional materials for sustainable energy and environmental applications.

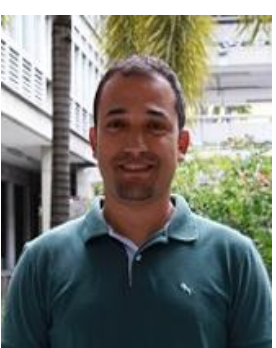

Henry A. Colorado is a Professor from Universidad de Antioquia, Colombia. He obtained his PhD and MSc in Materials Science and Engineering from University of California Los Angeles, and BSc and MSc from National University of Colombia. Henry is leading a research group working in materials science, composites, and waste management.

Publisher's Note Engineered Science Publisher remains neutral with regard to jurisdictional claims in published maps and institutional affiliations. 